\title{
The kinesin KIF1C transports APC-dependent mRNAs to cell protrusions
}

\author{
XAVIER PICHON, ${ }^{1,2,12}$ KONSTADINOS MOISSOGLU, ${ }^{3,12}$ EMELINE COLENO, ${ }^{1,2,4}$ TIANHONG WANG, ${ }^{3}$ \\ ARTHUR IMBERT, ${ }^{5,6,7}$ MARIE-CÉCILE ROBERT, ${ }^{1,2,4}$ MARION PETER, ${ }^{1,2}$ RACHA CHOUAIB, ${ }^{1,2,8}$ \\ THOMAS WALTER, ${ }^{5,6,7}$ FLORIAN MUELLER, ${ }^{9,10}$ KAZEM ZIBARA, ${ }^{8,11}$ EDOUARD BERTRAND, ${ }^{1,2,4}$ \\ and STAVROULA MILI ${ }^{3}$ \\ ${ }^{1}$ Institut de Génétique Moléculaire de Montpellier, University of Montpellier, CNRS, 34293 Montpellier, France \\ ${ }^{2}$ Equipe labélisée Ligue Nationale Contre le Cancer, University of Montpellier, CNRS, 34000 Montpellier, France \\ ${ }^{3}$ Laboratory of Cellular and Molecular Biology, Center for Cancer Research, National Cancer Institute, NIH, Bethesda, Maryland 20814, USA \\ ${ }^{4}$ Institut de Génétique Humaine, University of Montpellier, CNRS, 34396 Montpellier, France \\ ${ }^{5}$ MINES ParisTech, PSL-Research University, CBIO-Centre for Computational Biology, 77300 Fontainebleau, France \\ ${ }^{6}$ Institut Curie, 75248 Paris Cedex, France \\ ${ }^{7}$ INSERM, U900, 75248 Paris Cedex, France \\ ${ }^{8}$ Biology Department, Faculty of Sciences-I, Lebanese University, Beirut, Lebanon \\ ${ }^{9}$ Unité Imagerie et Modélisation, Institut Pasteur and CNRS UMR 3691, 75015 Paris, France \\ ${ }^{10} \mathrm{C} 3 \mathrm{BI}$, USR 3756 IP CNRS - Paris, France \\ ${ }^{11}$ ERO45, PRASE, DSST, Lebanese University, Beirut, Lebanon
}

\begin{abstract}
RNA localization and local translation are important for numerous cellular functions. In mammals, a class of mRNAs localize to cytoplasmic protrusions in an APC-dependent manner, with roles during cell migration. Here, we investigated this localization mechanism. We found that the KIF1C motor interacts with APC-dependent mRNAs and is required for their localization. Live cell imaging revealed rapid, active transport of single mRNAs over long distances that requires both microtubules and KIF1C. Two-color imaging directly revealed single mRNAs transported by single KIF1C motors, with the 3'UTR being sufficient to trigger KIF1C-dependent RNA transport and localization. Moreover, KIF1C remained associated with peripheral, multimeric RNA clusters and was required for their formation. These results reveal a widespread RNA transport pathway in mammalian cells, in which the KIF1C motor has a dual role in transporting RNAs and clustering them within cytoplasmic protrusions. Interestingly, KIF1C also transports its own mRNA, suggesting a possible feedback loop acting at the level of mRNA transport.
\end{abstract}

Keywords: RNA localization; local translation; RNA transport; cytoplasmic protrusions

\section{INTRODUCTION}

Localization of mRNA to specific subcellular compartments is an important mechanism for the spatio-temporal regulation of gene expression in diverse cell types and organisms (Chin and Lécuyer 2017; Eliscovich and Singer 2017). Subcellular mRNA localization allows localized protein synthesis and this is important for many biological functions such as cell fate determination (Berleth et al. 1988), cell polarization (Condeelis and Singer 2005), cell division (Chouaib et al. 2020; Ryder et al. 2020), cell migra-

\footnotetext{
${ }^{12}$ These authors contributed equally to this work.

Corresponding authors: edouard.bertrand@igh.cnrs.fr, voula.mili@nih.gov

Article is online at http://www.rnajournal.org/cgi/doi/10.1261/rna. 078576.120 . Freely available online through the RNA Open Access option.
}

tion (Katz et al. 2012; Wang et al. 2017; Moissoglu et al. 2020), embryonic patterning (Forrest and Gavis 2003), and synaptic plasticity (Martin and Zukin 2006; Lin and Holt 2007). One of the best characterized examples is the yeast Ash1 mRNA that localizes specifically in the bud of the daughter cells and encodes a transcriptional repressor protein involved in suppressing mating-type switching (Paquin and Chartrand 2008). Studies of this and other models revealed that the subcellular localization of mRNA relies on three main mechanisms, acting separately or in combination: random diffusion combined with local entrapment, general transcript degradation

(C) 2021 Pichon et al. This article, published in RNA, is available under a Creative Commons License (Attribution 4.0 International), as described at http://creativecommons.org/licenses/by/4.0/. 
coupled to localized protection and directed transport along the cytoskeleton (Medioni et al. 2012; Cody et al. 2013; Bovaird et al. 2018).

Active, motor-driven transport of mRNAs along the cytoskeleton is thus far the most common localization mechanism. It generally involves cis-acting elements, also called zipcodes, contained in the $3^{\prime}$ UTR sequence of the transcript. This is exemplified by the case of the $\beta$-actin mRNA in vertebrates, which accumulates at the leading edge of migrating cells and was among the first localized mRNAs discovered (Singer 1993). This mRNA contains a zipcode sequence recognized by the RNA Binding Protein ZBP1, allowing the transport of $\beta$-actin mRNAs in a motordriven manner along the cytoskeleton (Kislauskis et al. 1993; Oleynikov and Singer 2003; Condeelis and Singer 2005; Liao et al. 2015). Interestingly, transport of $\beta$-actin mRNA by ZBP1 involves both microtubules (MTs) and actin filaments (Fusco et al. 2003; Oleynikov and Singer 2003), as well as several motors that display some cell type and compartment specificity. Indeed, MYO5A and KIF5A interact with ZBP1 to transport $\beta$-actin mRNAs in dendrites and axons (Ma et al. 2011; Nalavadi et al. 2012), while Myosin IIB (MYH10) and KIF11, which directly binds ZBP1, regulate the transport of $\beta$-actin mRNAs in fibroblasts and during cell migration (Latham et al. 2001; Song et al. 2015).

In vertebrate systems, the motors involved in RNA transport have been investigated mostly in neuronal cells. Kinesin-1 (KIF5) was shown to associate with neuronal RNP granules and to be involved in their trafficking (Kanai et al. 2004). Kinesin-1 was also implicated in transport of myelin basic protein (MBP) mRNA in oligodendrocytes (Carson et al. 1997) as well as in shank1 mRNA transport in rat neurons (Falley et al. 2009). Kinesin-2 (KIF3A/B/ KAP3) can transport RNAs in vitro (Baumann et al. 2020), but its in vivo relevance is still unclear. The involvement of additional motors, and the means through which they connect to potential RNA cargoes are still largely unexplored, especially in the case of nonneuronal cell types (Gagnon and Mowry 2011; Xing and Bassell 2013).

Localized RNAs are prevalent in nonneuronal, mesenchymal cells. Apart from $\beta$-actin, numerous other RNAs are localized at protrusions of mesenchymal cells and their local translation is important for cell migration (Mili et al. 2008; Mardakheh et al. 2015; Costa et al. 2020; Moissoglu et al. 2020). Localization of these RNAs is carried out through at least two distinct pathways. Specifically, a subset of about a hundred RNAs, which include transcripts encoding signaling and cytoskeleton regulators (such as the Rab GTPase RAB13, the RhoA exchange factor NET1, the collagen receptor DDR2, the motor related proteins TRAK2, DYNLL2, and others), require the APC tumor suppressor protein for localization and have been referred to as APC-dependent (Wang et al. 2017). Other protrusion-enriched RNAs, exemplified by RNAs encoding ribosomal proteins, do not require APC and exhibit distinct regulation (Wang et al. 2017).
Similar to what has been described for other localized RNAs, sequences within the $3^{\prime}$ UTR of APC-dependent RNAs are necessary and sufficient for targeting to the cell periphery (Mili et al. 2008). Specifically, interfering with or deleting particular GA-rich regions is sufficient to disrupt peripheral localization and perturb cell movement in various systems (Chrisafis et al. 2020; Costa et al. 2020; Moissoglu et al. 2020). Furthermore, localization to the periphery requires the microtubule cytoskeleton and in particular a subset of stable, detyrosinated microtubules (Wang et al. 2017; Moissoglu et al. 2019). Indeed, at least some APCdependent RNAs exhibit a colocalization with the plus ends of detyrosinated microtubules (Mili et al. 2008). The peripheral complexes also contain APC, a protein that has the ability to directly bind microtubules via its carboxyl terminus (Munemitsu et al. 1994; Zumbrunn et al. 2001; Jimbo et al. 2002; Barth et al. 2008; Bahmanyar et al. 2009), hence suggesting that APC might mediate the interaction of localized mRNAs with microtubules (Mili et al. 2008; Preitner et al. 2014).

An additional feature integrated with the localization of APC-dependent RNAs is their existence in distinct physical states. In particular, RNAs in internal or peripheral, actively extending cytoplasmic regions exist as single molecules that are undergoing translation. However, at some peripheral areas, single RNAs coalesce in multimeric heterogeneous clusters that are composed of multiple distinct RNA species. Interestingly, these clusters preferentially form at retracting protrusions and contain translationally silent mRNAs (Moissoglu et al. 2019). These data indicate the existence of a dynamic regulatory mechanism during cell migration, which coordinates local mRNA translation with protrusion formation and retraction. However, the exact mechanisms and molecular players involved in transport to the periphery and cluster formation for this group of RNAs are still unclear.

In this study, we focused on the kinesin KIF1C, which we recently showed to accumulate and colocalize with its own mRNA in cytoplasmic protrusions (Chouaib et al. 2020). We show here that KIF1C associates with additional protrusion-localized RNAs belonging to the APC-dependent group. We describe a specific mRNA transport mechanism by which the KIF1C kinesin motor binds APC-dependent mRNAs, including its own, actively transports them to cell protrusions in a $3^{\prime}$ UTR dependent manner and additionally participates in promoting and/or maintaining their peripheral clusters.

\section{RESULTS}

\section{Identification of a specific mRNA subset associating with the KIF1C motor in human cells}

High-throughput mRNA-protein cross-linking approaches previously showed that KIF1C directly binds mRNAs 
(Baltz et al. 2012; Castello et al. 2012), and we recently showed that KIF1C mRNAs and proteins colocalize together in protrusions of HeLa cells (Fig. 1A; Chouaib et al. 2020), suggesting that the KIF1C kinesin might be somehow involved in the metabolism of protrusion mRNAs. To determine the identity of the mRNAs bound by the KIF1C motor, we used a HeLa cell line stably expressing a KIF1C-GFP fusion from a bacterial artificial chromosome containing all the regulatory sequences of the human KIF1C gene, including its $5^{\prime}$ and $3^{\prime}$ UTRs (Poser et al. 2008; Chouaib et al. 2020). We immunoprecipitated (IP) KIF1C-GFP with anti-GFP antibodies or uncoated beads as controls, and identified the coprecipitated RNAs using microarrays (Fig. 1B; Supplemental Table S1). We found that many mRNAs were enriched in the KIF1C-GFP IP as compared to the control IP. To explore in more detail the localization of the mRNAs associated with KIF1C, we performed a small smFISH localization screen in HeLa cells. We tested the 26 most enriched mRNAs in the KIF1C IP (Supplemental Tables S1, S2) and found four that were peripherally enriched. These included the RAB13 mRNA (5.7fold enrichment, Supplemental Table S1), along with the KIF1C mRNA itself (2.6-fold enrichment) and the NET1 and TRAK2 mRNAs (5.7- and 4.9-fold enrichment, respectively). We had previously reported that these transcripts localize to protrusions of mouse cells in an APC-dependent manner (Wang et al. 2017), and we thus focused on them. Visual examination of the images revealed that KIF1C, NET1, TRAK2, and RAB13 mRNAs clearly localized also to protrusions of HeLa cells (Supplemental Fig. S1A). To confirm the link between APC-dependent mRNA localization and binding to KIF1C protein, we performed a correlation analysis of the two metrics (Supplemental Fig. S1B; Supplemental Table S3). This indicated that APC-dependent mRNAs indeed preferentially associate with KIF1C protein, while mRNAs coding for ribosomal proteins, which often localize to protrusions independently of APC (Wang et al. 2017), do not. The IP/microarray data thus show a physical link between KIF1C and mRNAs that localize to protrusions in an APC-dependent manner.

Next, we tested whether these mRNAs colocalize with the KIF1C protein in vivo. To this end, we performed smFISH experiments in a HeLa cell line stably expressing a KIF1C-GFP mRNA from a cDNA and found that indeed, KIF1C, NET1, TRAK2, and RAB13 mRNAs colocalized with the KIF1C-GFP protein in cytoplasmic protrusions (Fig. 1C). In order to show that this colocalization reflected a molecular interaction, we performed single-molecule imaging using the SunTag system (Tanenbaum et al. 2014). To this end, we generated a stable HeLa cell line expressing KIF1C-fused to 24 repeats of the GCN4 epitope (KIF1CSunTag $\left.{ }_{\times 24}\right)$, together with the single-chain variable fragment fused to sfGFP (scFv-sfGFP). This system enables the detection of single molecules of the KIF1C protein (Fig. 1D; Tanenbaum et al., 2014). We thus combined detection of single KIF1C-SunTag $\times 24$ proteins with single mRNA detection by smFISH, using probes against RAB13 and NET1 mRNA, or CRM1 and RBP1 mRNAs as controls (Fig. 1D,E; Supplemental Fig. S1C-E). KIF1C-SunTag×24 proteins were found to colocalize with RAB13 and NET1 mRNAs at protrusions as expected, while the control mRNAs did not. In addition, we also observed colocalization of KIF1C-SunTag $\times 24$ with both RAB13 and NET1 mRNAs at the single-molecule level at more internal locations in the cytoplasm (Fig. 1E, panels 1, 3,4; Supplemental Fig. S1D). This confirmed the interaction of single molecules of KIF1C protein with single molecules of RAB13 mRNAs. Taken together, these data raise the possibility that the kinesin KIF1C might be part of a mechanism that localizes APC-dependent mRNAs to cytoplasmic protrusions.

\section{KIF1C associates with APC and is required for the localization of APC-dependent mRNAs to cytoplasmic protrusions in human and mouse cells}

To further support the connection between KIF1C and APC-dependent RNAs we tested whether KIF1C and APC interact in cells. Indeed, immunoprecipitation of GFP-APC revealed a specific association with KIF1CmCherry (Fig. 2A). We additionally imaged the two fluorescent proteins in live cells and detected a colocalization between GFP-APC and KIF1C-mCherry in peripheral clusters (Fig. 2B). Therefore, KIF1C exhibits a specific association with both APC protein and peripherally localized APC-dependent RNAs.

To test whether the localization of APC-dependent mRNAs in protrusions depended on the KIF1C protein, we depleted KIF1C by multiple siRNAs in HeLa or MDAMB-231 cells, and performed smFISH experiments using probes against RAB13 or NET1 mRNAs. Intracellular distributions of mRNAs were quantitatively assessed by calculating a Peripheral Distribution Index (PDI), a metric that distinguishes diffusely distributed from peripherally localized RNAs, by measuring the distance of the RNA signal relative to the centroid of the nucleus (Wang et al. 2017; Stueland et al. 2019). For each cell, the RNA distribution is normalized to a hypothetical uniform distribution such that a PDI value of 1 indicates a uniform, diffuse signal, while values smaller or greater than 1 indicate a perinuclear or peripheral localization, respectively. Remarkably, both RAB13 and NET1 mRNAs became less localized when KIF1C expression was reduced with siRNAs (Supplemental Fig. S2A-D), demonstrating that the KIF1C kinesin was required for mRNA localization in human cells.

Next, we moved to a mouse system, NIH/3T3 cells, where the localization of mRNAs in protrusions has been extensively studied (Chicurel et al. 1998; Mili et al. 2008; Wang et al. 2017; Moissoglu et al. 2019). To test whether the KIF1C protein has a general role in localizing mRNAs 
A Human HeLa cells

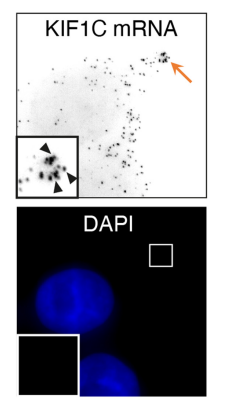

C
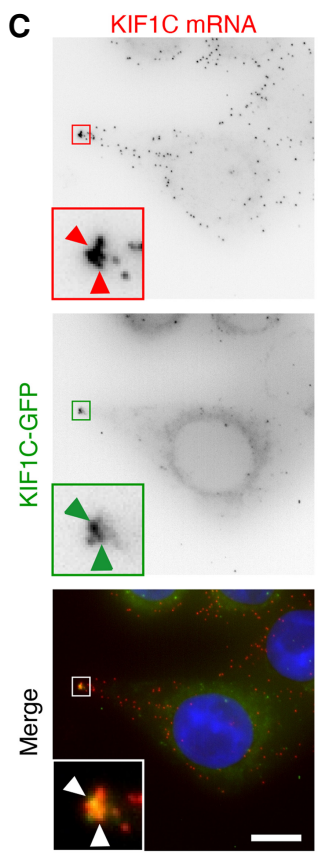

D

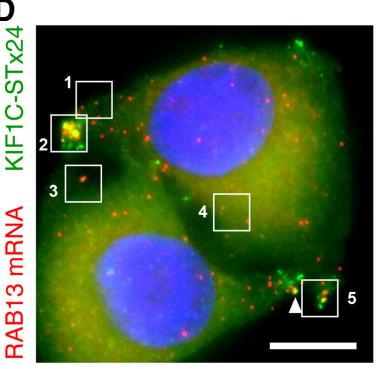

B
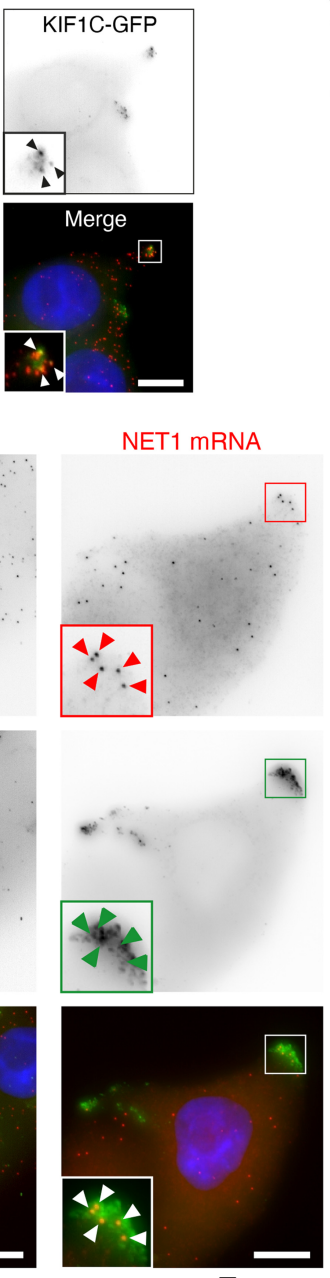

$E$

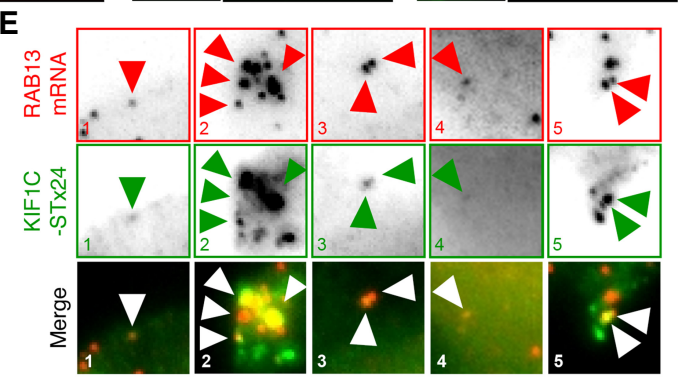

FIGURE 1. Identification of mRNAs associated with the KIF1C motor. (A) The KIF1C kinesin colocalizes with its mRNA in protrusions of HeLa cells. Images are micrographs of a H9 Flipln HeLa cell line stably expressing a KIF1C-GFP cDNA. (Top left) KIF1C mRNA detected by smFISH with probes against the endogenous mRNA; (top right) KIF1C-GFP protein; (bottom left) DNA stained with DAPI; (bottom right) merge of the two signals with the KIF1C-GFP protein in green and KIF1C mRNA in red. Orange arrow: a cell protrusion. Blue: DNA stained with DAPI. Scale bar: 10 microns. Insets represent zooms of the boxed areas in the merge panel. White and black arrowheads indicate the colocalization of KIF1C mRNA with KIF1C-GFP protein. (B) Transcripts associating with the KIF1C-GFP protein. The graph depicts the microarray signal intensity of RNAs detected in a KIF1C-GFP pull-down (x-axis), versus the control IP ( $y$-axis). Each dot represents an mRNA. Red dot: KIF1C mRNA; blue dot: mRNAs enriched in the KIF1C-GFP IP. N=2. (C) Colocalization of KIF1C-GFP with KIF1C, NET1, TRAK2, and RAB13 mRNAs. Images are micrographs of HeLa H9 Flip-In cells stably expressing a KIF1C-GFP cDNA, labeled by smiFISH with probes against the indicated mRNAs. (Top) Cy3 fluorescent signals corresponding to endogenous KIF1C, NET1, TRAK2, and RAB13 mRNAs. (Middle) KIF1C-GFP signal. (Lower) Merge with the Cy3 signal in red and GFP signal in green. Blue: DNA stained with DAPI. Scale bar: 10 microns. Arrowheads indicate accumulation of single mRNA molecules at cell protrusions. (D) Single-molecule colocalization of KIF1C-ST-x24 with RAB13 mRNAs. Images are micrographs of Hela cells stably expressing KIF1C-STx24 and scFv-GFP. Red: Cy3 fluorescent signals corresponding to RAB13 mRNAs labeled by smiFISH with probes against endogenous RAB13 mRNA. Green: GFP signal corresponding to single molecules of KIF1C protein. Blue: DNA stained with DAPI. Scale bar: 10 microns. (E) Insets represent zooms of the numbered areas from panel $D$. Legend as in D. Arrowheads indicate molecules of RAB13 mRNA and KIF1C-STx24 protein. Micrographs show cells representative of the population. 


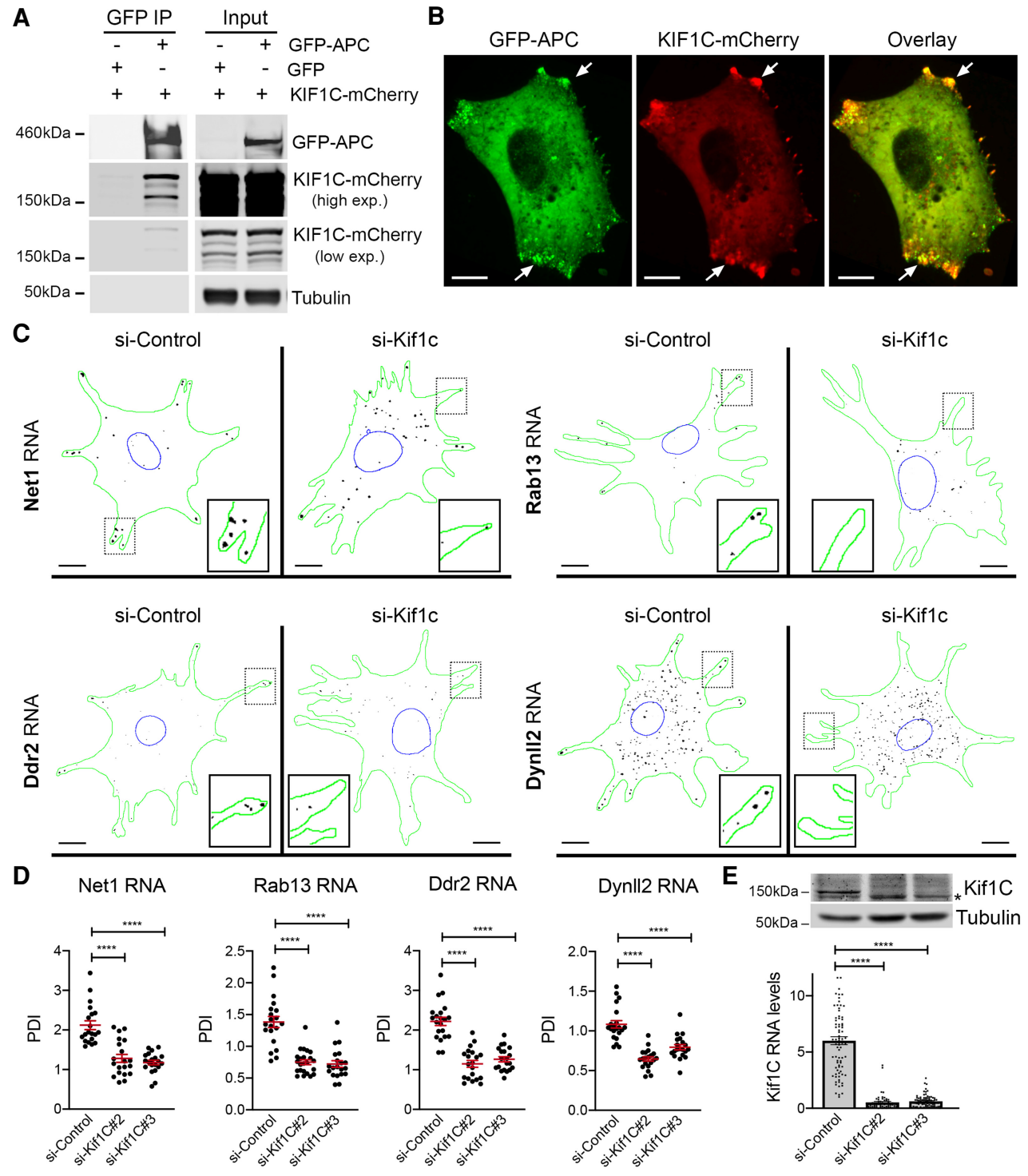

FIGURE 2. KIF1C associates with $A P C$ and is required for the localization of APC-dependent mRNAs to cytoplasmic protrusions. (A) Coimmunoprecipitation of KIF1C with APC. GFP or GFP-APC were immunoprecipitated from cells also expressing KIF1C-mCherry and analyzed by western blot to detect the indicated proteins. Results are representative of three independent experiments. (B) Colocalization of GFP-APC and KIF1C-mCherry at cytoplasmic protrusions (arrows). Images are representative of multiple cells observed in two independent experiments. Scale bar: 10 microns. (C) Depletion of Kif1c prevents mRNA accumulation in protrusions. Images are micrographs of NIH/3T3 cells labeled by smFISH with probes against Net1, Rab13, Ddr2, and Dynll2 mRNAs, following treatment with siRNAs against Kif1C (panels si-Kif1c) or a control sequence (panel si-Control). Scale bars: 10 microns. Green: outline of the cells; blue: outline of the nuclei; black: smFISH signals. Insets represent magnifications of the boxed areas. (D) Quantification of mRNA localization of cells described in C. Graphs represent the intracellular distribution of the indicated mRNAs as measured by PDI index, with and without treatment of cells with the indicated siRNAs. Red bars represent the mean and $95 \%$ confidence interval. Points indicate individual cells observed in two independent experiments. (E) Detection of Kif1C protein (upper panels) or Kif1C RNA levels (lower graph) from cells treated with the indicated siRNAs. Stars in $D$ and $E$ are $P$-values: $(* * * *) P<0.0001,\left({ }^{* * *}\right) P<0.001$, estimated by analysis of variance with Bonferroni's multiple comparisons test.

at cell protrusions, we assessed the localization of a series of APC-dependent and APC-independent mRNAs by smFISH, following depletion of KIF1C expression with two different siRNAs. As shown in Figure $2 \mathrm{C}-\mathrm{E}$ and Supplemental Figure S2E,F, several APC-dependent RNAs, including Net1, Rab13, Ddr2, Dynll2, and Cyb5r3, exhibited a protrusion localization pattern that was lost fol- lowing KIF1C depletion. Indeed, KIF1C loss led to RNA distributions that were mostly diffuse (PDI values centering around 1; Fig. 2B; Supplemental Fig. S2F), indicating that the KIF1C motor has an important contribution toward directing peripheral mRNA localization. Interestingly, the localization of two APC-independent mRNAs, Rps20 and Rpl27a, was not affected (Supplemental Fig. S2G). To 
ascertain that this effect was not due to altered mRNA expression, we measured their levels following KIF1C depletion (Supplemental Fig. S2H). This analysis showed no changes in the overall abundance of APC-dependent mRNAs, except for the depleted KIF1C mRNA (Supplemental Fig. S2H). Therefore, we conclude that KIF1C is required for the localization of APC-dependent mRNAs to cell protrusions, in various human and mouse cells.

\section{KIF1C is required for active transport of APC- dependent mRNAs on microtubules}

To monitor trafficking of APC-dependent mRNAs, we expressed in NIH/3T3 fibroblasts a reporter carrying the $\beta$ globin coding sequence followed by 24 binding sites for the bacteriophage MS2 coat protein (MCP; Fig. 3A schematic). Binding of coexpressed MCP-GFP to these sites allows visualization and tracking of single molecules of the reporter mRNA in living cells (Fusco et al. 2003). To recapitulate the localization of APC-dependent RNAs, the reporter additionally included a control 3'UTR or the 3'UTR of Net1 or Rab13 (hereafter referred to as $\beta 24 \mathrm{bs} / \mathrm{Ctrl}$, $\beta 24 \mathrm{bs} / \mathrm{Net1}$, and $\beta 24 \mathrm{bs} /$ Rab13, respectively). As shown previously, these $3^{\prime}$ UTR sequences are sufficient to direct peripheral distribution of this reporter transcript in $\mathrm{NIH} /$ 3 T3 cells (Moissoglu et al. 2019). We initially examined trafficking of the reporter during early stages of cell spreading, which mimic conditions in actively protruding cell regions. Indeed, live fluorescence imaging of the reporter containing the Net1 3'UTR revealed a distinct peripheral pattern after plating cells on fibronectin for $30 \mathrm{~min}$ (Fig. 3A; Supplemental Movie S1). Because kinesin-dependent mRNA trafficking is expected to occur on the microtubule cytoskeleton, it was important to identify microtubule-dependent events and discriminate them from other modes of motion. For this, reporter particles were tracked in cells before and after 15 min of nocodazole treatment. To identify long and linear movements, as those expected to occur on microtubules, we used two different metrics to quantitatively describe individual tracks: "Linearity of forward progression" and "Track displacement." To determine the range of these parameters that define directed microtubule-based tracks, we compared movements before and after microtubule depolymerization. From this, we separated "long/directed" tracks (Fig. 3B). These tracks exhibit higher displacement and linearity (net displacement $>4$ microns, linearity $>0.7$ ), and they are absent in cells treated with nocodazole. They correspond to $~ 3 \%-6 \%$ of the total tracks (Fig. 3B; Supplemental Movies S1, S2). Consistent with their representing persistent directed motions, this subset of tracks exhibit high Mean Square Displacements (MSD) over their lifetime (generally more than $15 \mu^{2}$ ) and display positive velocity autocorrelation (Fig. 3C, left panels). In contrast, the remaining tracks, which we classify as "short/diffuse," exhibit characteristics similar to tracks of nocodazole-treated cells. Specifically, they exhibit low MSDs and zero velocity autocorrelation, as is characteristic of diffusive Brownian motions (Fig. 3C, middle and right panels). Both "long/directed" and "short/diffuse" tracks have a similar range of lifetimes, while "long/directed" tracks have a higher mean speed (Supplemental Fig. S3).

Tracking of a reporter carrying the Rab13 3'UTR also exhibited long/directed tracks with dependence on microtubules (Fig. 3D,E). In contrast, a control reporter lacking a localizing 3'UTR did not produce tracks with these characteristics (Fig. 3D,E). Importantly, this subset of tracks was not affected by disruption of the actin cytoskeleton with cytochalasin $\mathrm{D}$ or following treatment with a control vehicle, DMSO (Fig. 3F; Supplemental Fig. S4; Supplemental Movies S3, S4). Thus, the reporters carrying the 3'UTR of Net1 or Rab13 mimic the localization pattern of APC-dependent mRNAs and allow the identification of long and linear microtubule- and 3'UTR-dependent transport events.

To directly test the role of KIF1C in these trafficking events, we visualized fluorescent particles of the reporter carrying the Net1 3'UTR and measured the frequency of long, directed microtubule-dependent displacements in actively spreading cells following KIF1C depletion. As previously observed with endogenous transcripts (Fig. 2), reporter mRNAs became less localized when KIF1C expression was reduced with siRNAs (Fig. 4A; Supplemental Movies S5-S8). Importantly, track analysis showed that KIF1C loss significantly reduced the number of the microtubule-dependent displacements (Fig. 4B,C). To assess specificity, we depleted two additional kinesins that have been linked to APC and RNA transport, KIF5B and KIF3A (Kanai et al. 2004; Dunn et al. 2008; Cai et al. 2009; Yasuda et al. 2017; Baumann et al. 2020). Figure 4A-C shows that depleting these kinesins did not change the overall peripheral accumulation of the reporter and did not result in a reduction of the long, directed transport events. Thus, KIF1C exhibits a specific function in transporting APC-dependent mRNAs via microtubules in actively protruding cell regions.

\section{Peripheral clustering of APC-dependent mRNAs depends on KIF1C}

Peripheral APC-dependent mRNAs can form large heterogeneous clusters that are translationally silent (Moissoglu et al. 2019). These clusters often associate with retracting protrusions in migrating cells, suggesting that they are part of a spatio-temporal control of protein synthesis (Moissoglu et al. 2019). Formation of these clusters is recapitulated by the reporter constructs carrying the Net1 or Rab13 3'UTR, but not by a control reporter (Supplemental Fig. S5). These clusters are visible at later time points after plating (approximately $3 \mathrm{~h}$ ), when most protrusions are not actively extending, consistent with the appearance of endogenous RNA clusters in nonextending or retracting 
A

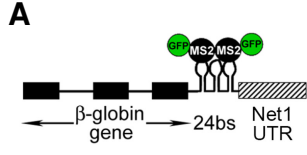

Plating for 30min

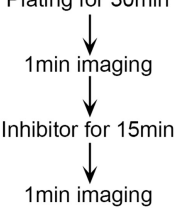

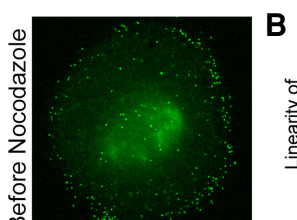

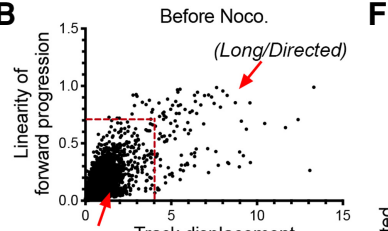

(Short/Diffuse) Track displacement
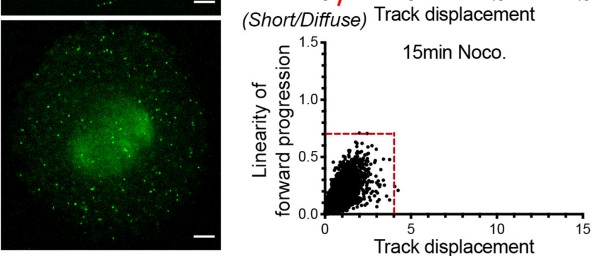

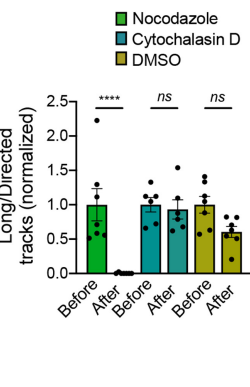

C
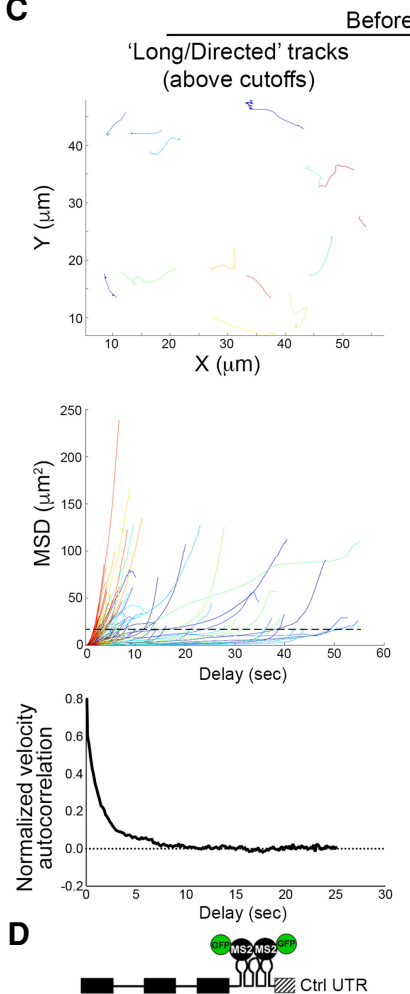

\section{s} (below cutoffs)
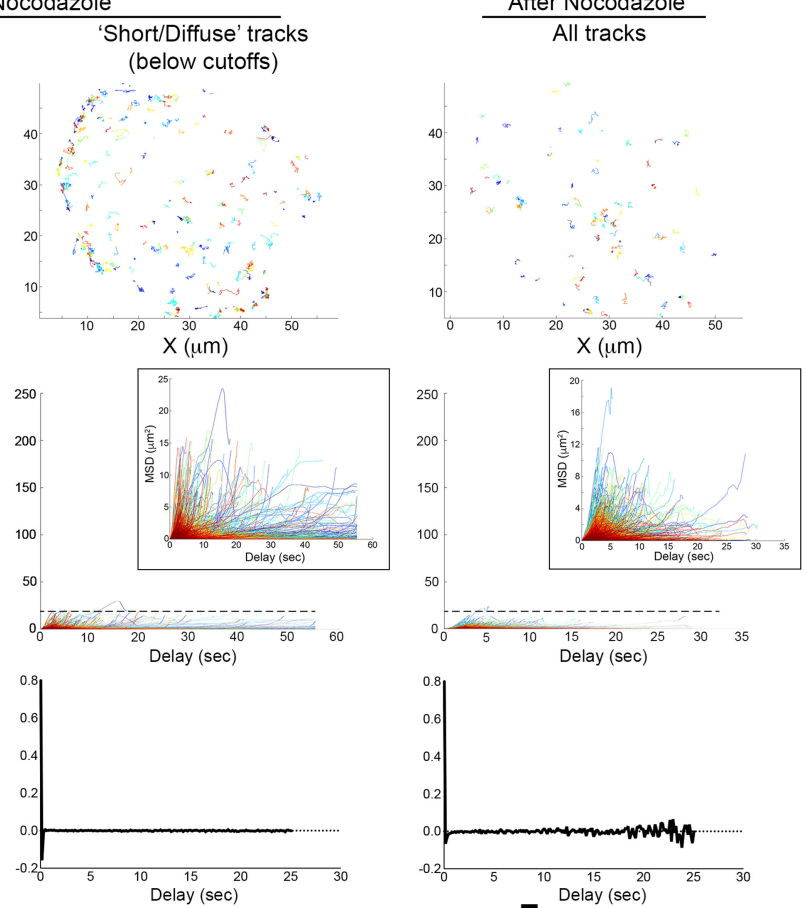

बruszins?

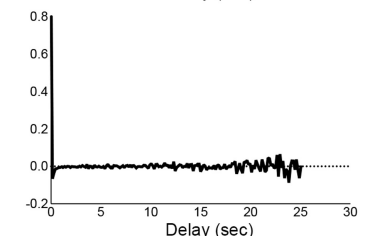

E

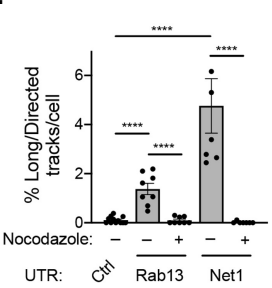

FIGURE 3. Reporter mRNAs containing Net1 and Rab13 3'UTRs display long, directed microtubule-dependent displacements. (A) Schematic of the mRNA reporter construct containing the $\beta$-globin coding sequence followed by $24 x M S 2$ binding sites and the mouse Net $13^{\prime} \mathrm{UTR}$ ( $\beta 24 \mathrm{bs} / \mathrm{Net} 1$ ). Images are snapshots of live $\mathrm{NIH} / 3 \mathrm{~T} 3$ before or after nocodazole treatment, following the experimental scheme detailed on the left. High speed imaging was performed over 1 min to track individual RNA movements. See Supplemental Movies S1, S2 for time lapse imaging. The cells stably expressed the $\beta 24 \mathrm{bs} /$ Net1 reporter mRNA and MCP-GFP (Green). Scale bar: 5 microns. (B) The graphs plot the displacement over the linearity of forward progression (defined as the mean straight line speed divided by the mean speed) of single RNA tracks from cells treated as described in $A$. Red lines indicate the thresholds used to separate "long/directed" from "short/diffuse" tracks. N=6-7 cells. (C) Analysis of "long/directed" or "short/diffuse" tracks, from untreated cells, or of all tracks from nocodazole-treated cells, from B. Individual raw tracks from a representative cell are displayed in the upper panels. Mean square displacement (MSD) (middle panels) and velocity autocorrelation (bottom panels) of tracks are shown. $N=180$ (long/directed); 4427 (short/diffuse); 3795 (after nocodazole) tracks from seven cells. Note that "long/directed" tracks exhibit higher MSD (>15 $\mu \mathrm{m}^{2}$, dashed black line), than "short/diffuse" or nocodazole-treated tracks, as well as positive velocity autocorrelation. Insets of MSD plots present zooms of $y$-axis scale. (D) Single RNA molecules of the $\beta 24 \mathrm{bs} /$ Control $3^{\prime} U T R$ (Ctrl) or the $\beta 24 \mathrm{bs} /$ Rab13 $3^{\prime} U T R$ reporters were tracked over $1 \mathrm{~min}$ period in cells treated or not with nocodazole. Graphs plot the displacements of individual tracks ( $x$-axis) over the linearity of their forward progression ( $y$-axis). Red lines indicate the thresholds used to filter tracks of molecules undergoing directed movement. $N=8-12$ cells. $(E)$ The graph depicts the percentage of long/directed tracks of the indicated reporters per cell following treatment with nocodazole. Stars represent $P$-values: $(* * *) P<0.0001$, estimated using one-way analysis of variance with Sidak's multiple comparisons test. Error bars: standard error of the mean. $(F)$ The bar plot depicts the percentage of long/directed tracks per cell before and after treatment with the indicated compounds. Average values of respective "Before" values were set to $1 . N=6-7$. Stars represent $P$-values: $\left(^{* * * *}\right) P<0.0001$, ns: nonsignificant, estimated using one-way analysis of variance with Tukey's multiple comparisons test. Error bars: standard error of the mean. 

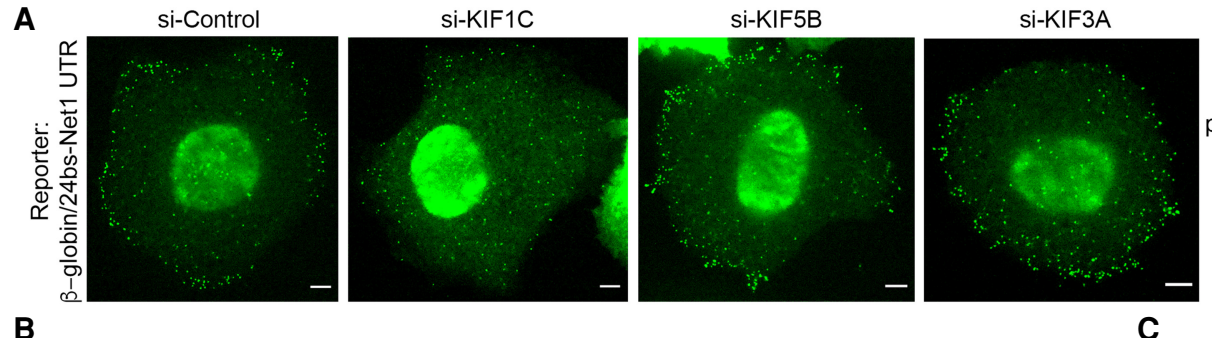

$\mathrm{NIH} / 3 \mathrm{~T} 3$ cells

plating for $30 \mathrm{~min}$

B
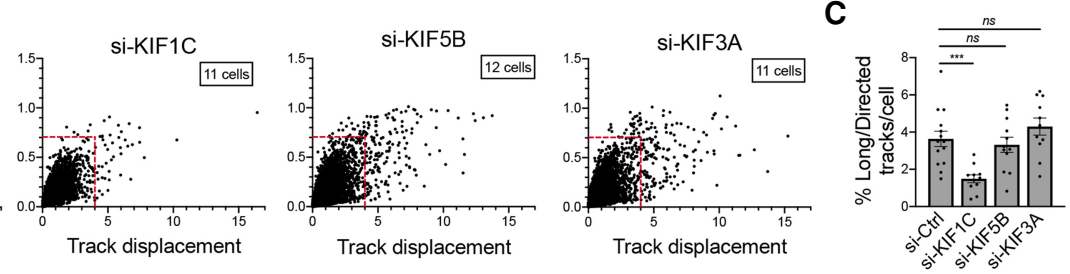

FIGURE 4. Reporter mRNAs containing the Net1 $3^{\prime}$ UTR require the Kif1c motor for long, linear microtubule-dependent displacements. (A) Images are snapshots of live NIH/3T3 cells taken 30 min after plating. The cells stably expressed the $\beta 24 \mathrm{bs} / \mathrm{Net} 1 \mathrm{mRNA}$ reporter and MCPGFP (Green) and were treated with the indicated siRNAs. The green spots correspond to single mRNAs detected with the MCP-GFP. High speed imaging was performed over 1 min to track individual RNA movements. See Supplemental Movies S5-S8 for time lapse imaging. Scale bars are 5 microns. (B) Graphs plot the displacements of individual RNA tracks ( $x$-axis) over the linearity of their forward progression ( $y$-axis) (defined as the mean straight line speed divided by the mean speed), using the movies of cells as shown in A. Red lines indicate the thresholds used to filter tracks of molecules undergoing directed movement (based on Fig. 3). (C) Graph depicts the percentage of directed tracks per cell following treatment with the indicated siRNAs (see panel B). $N=11-14$ cells. Stars represent $P$-values: $\left(^{* * *}\right) P<0.001$, ns: nonsignificant, estimated using one-way analysis of variance with Dunnett's multiple comparisons test. Error bars: standard error of the mean.

protrusions (Fig. 5A; Moissoglu et al., 2019). These clusters can be identified as bright particles with intensities higher than those characteristic of single molecules (Fig. 5A). To test whether KIF1C is implicated in the formation of these clusters, we scored the frequency of bright particles in KIF1C-depleted cells during late stages of spreading ( $3 \mathrm{~h}$; Fig. 5B,C; particle brightness $>4950$ ). As shown in Figure 5 , while clusters formed by the Net1 3'UTR-reporter were readily observed in protrusions of control siRNA-treated cells, their frequency was substantially reduced, and mostly single molecules were present, when KIF1C was depleted (Fig. 5A-C). Moreover, cluster formation was only marginally affected by the depletion of KIF5B or KIF3A. Thus, KIF1C specifically controls the clustering of APC-dependent mRNAs. We note that clusters are not detected even in protrusions containing a substantial amount of single RNA molecules (see enlarged KIF1C insets in Fig. 5A), suggesting that cluster loss is not a secondary consequence of a reduced number of mRNA molecules arriving at protrusions upon KIF1C depletion. We rather think that these results indicate an additional role of KIF1C in forming higher order RNP complexes at protrusions.

\section{Single-molecule two-color imaging provides direct evidence that the KIF1C motor transports protrusion mRNAs}

To provide direct evidence that the protrusion mRNAs are transported by the KIF1C motor, we performed two-color single-molecule imaging of mRNAs and motors, in order to visualize cotransport of the two types of molecules. To this end, we used the NIH/3T3 cells expressing the Net1 3'UTR-containing reporter and modified them to also stably express a KIF1C protein fused to the SunTag (KIF1C$\mathrm{ST}_{\times 24}$ ), together with a single-chain variable fragment antibody fused to $\mathrm{mScarletl}$ (scFv-mScarletl).

Imaging of fixed cells showed that the KIF1C-SunTag ${ }_{x 24}$ motor and reporter mRNAs accumulated in protrusions as expected (Fig. 6A, B; Supplemental Fig. S4A,B). Moreover, we could also occasionally detect colocalization events where a single molecule of KIF1C-SunTag ${ }_{\times 24}$ would colocalize with a single reporter mRNA at internal cellular areas. To confirm that this colocalization was relevant to mRNA transport, we performed two-color live-cell imaging using movies recorded at a high frame rate $(7.36 \mathrm{fps}$ for $52 \mathrm{sec}$ ). This allowed the detection of cotransport events, in which a single molecule of KIF1C-ST ${ }_{\times 24}$ moved with a reporter mRNA molecule in a rectilinear manner at high speed (Fig. 6C,D; Supplemental Movie S9; Supplemental Fig. S4C,D; Supplemental Movie S10). Kymographs confirmed that both molecules moved together in an anterograde direction toward protrusion (Fig. 6E; Supplemental Fig. S4E), traveling an average distance of 22 microns at speeds of 2.6 $\mu \mathrm{m} / \mathrm{sec}$ (Fig. 6F,G). Taken together, these data demonstrate that the KIF1C kinesin actively transports this Net1 reporter mRNA to cell protrusions along microtubule cables.

\section{DISCUSSION}

RNA transport along the cytoskeleton is a well-established mechanism allowing subcellular mRNA localization and local translation. In mammals, a large class of mRNAs localize 


\section{A}

A $\quad \beta$-globin/24xMS2bs-Net1 UTR
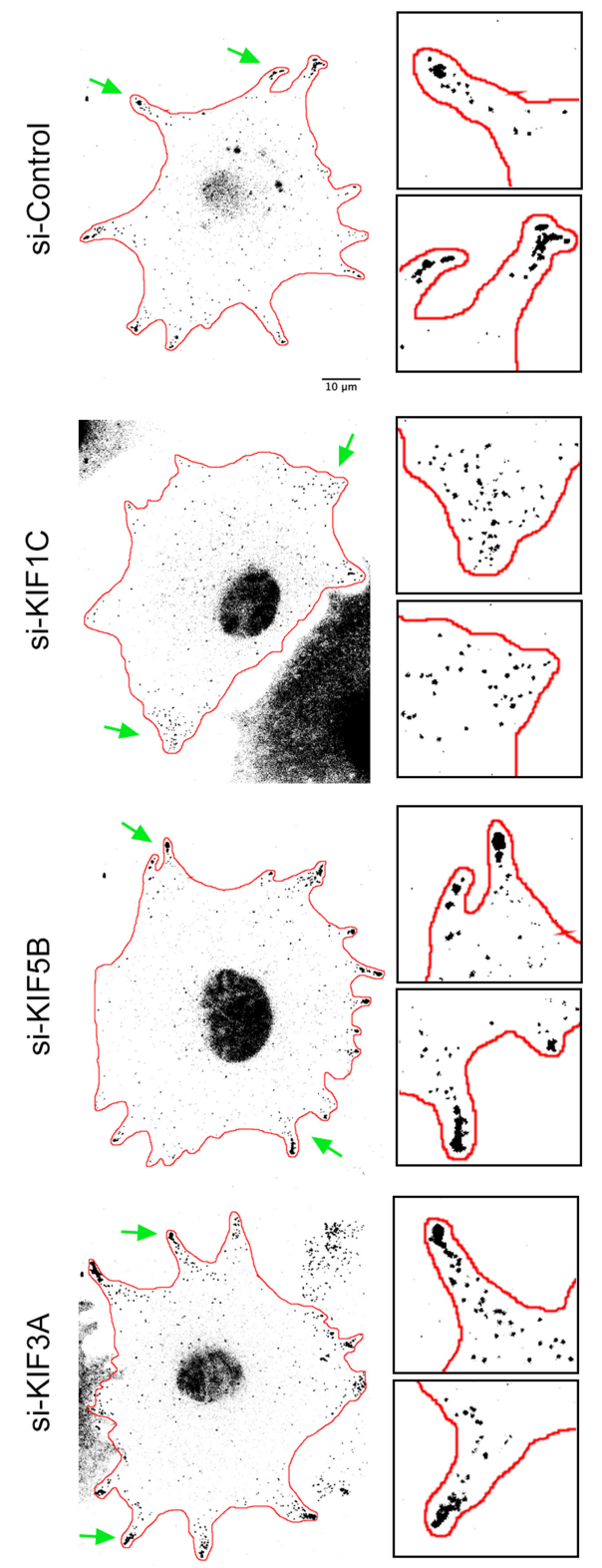

$\mathrm{NIH} / 3 \mathrm{~T} 3$ cells

3hrs plating
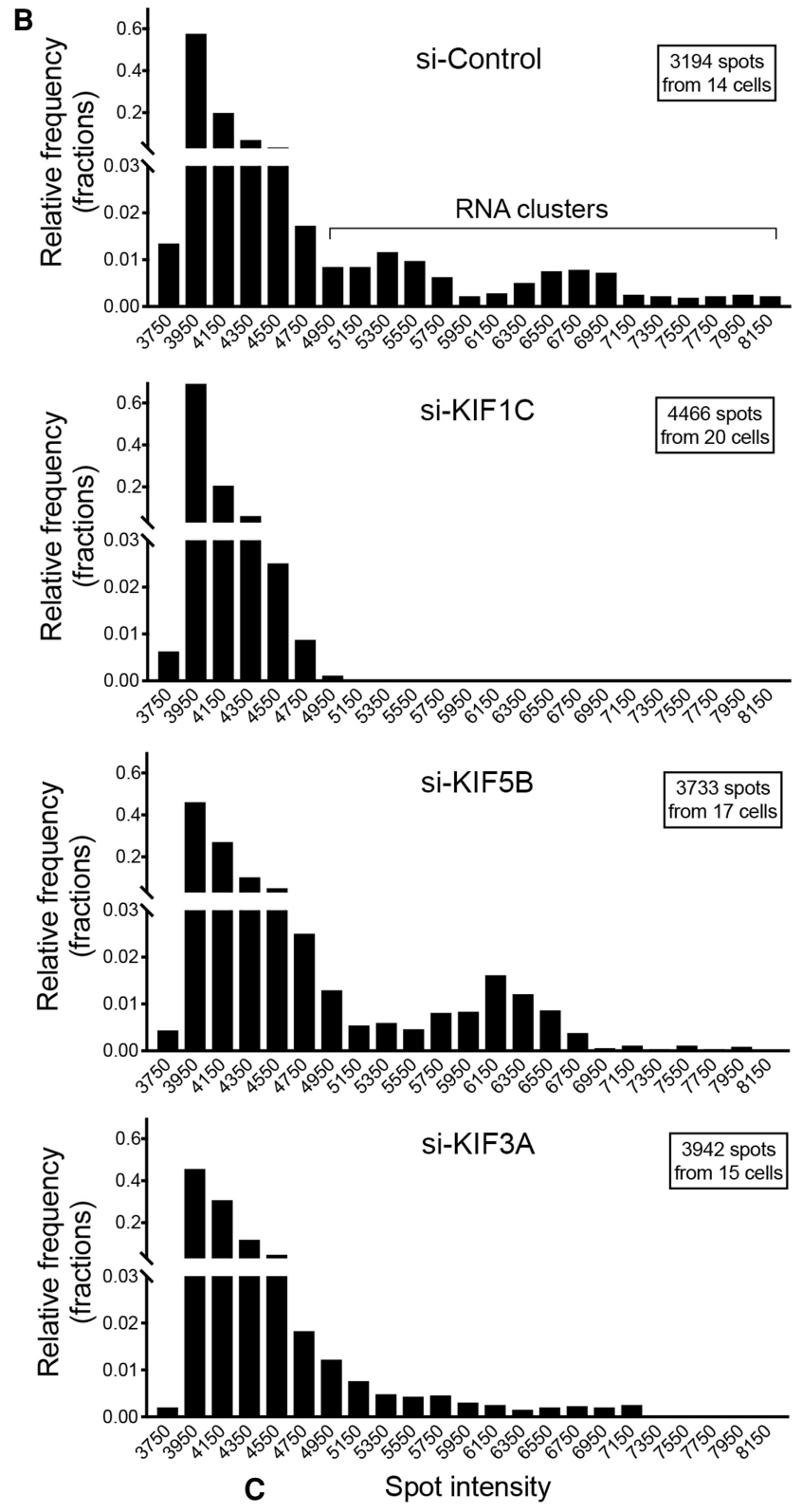

C

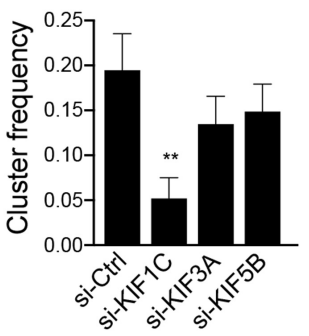

FIGURE 5. Kif1c is required for the peripheral clustering of reporter mRNAs containing the Net1 $3^{\prime} U T R$ in live mouse fibroblasts. (A) Images are micrographs of live NIH/3T3 cells taken $3 \mathrm{~h}$ after plating and expressing MCP-GFP and the $\beta 24 \mathrm{bs} / \mathrm{Net} 1$ reporter mRNA. Cells were treated with the siRNAs indicated on the left. Scale bar is 10 microns. Boxed insets are magnifications of the areas indicated by green arrows. (B) Frequency histograms of the intensities of the $\beta 24 \mathrm{bs} /$ Net1 reporter mRNA spots following treatment with the indicated siRNAs, measured from images as shown in $A$, from $N=14-20$ cells. The majority of molecules fall under a single lower intensity peak, likely indicative of single molecules, while a smaller fraction exhibits higher intensities indicative of higher order clusters. (C) Graph depicts the mRNA cluster frequency per cell following treatment of cells with the indicated siRNAs. Clusters correspond to $\beta 24 \mathrm{bs} / \mathrm{Net} 1 \mathrm{mRNA}$ spots of intensities higher than 4950 , measured from the graphs shown in $B . N=14-20$ cells. Stars represent $P$-values: $\left(^{* *}\right) P<0.01$, estimated using one-way analysis of variance with Dunnett's multiple comparisons test. Error bars: standard error of the mean. 


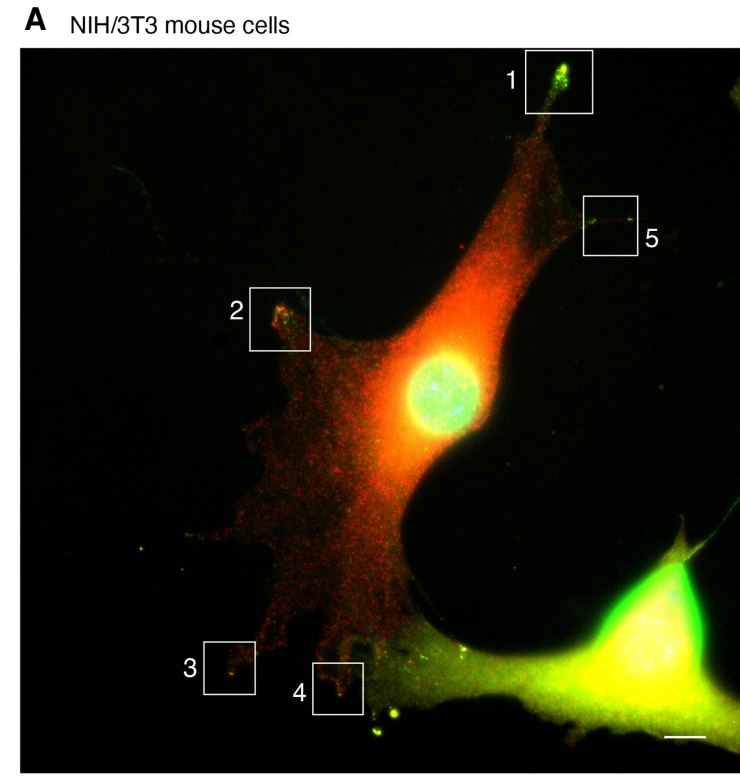

C

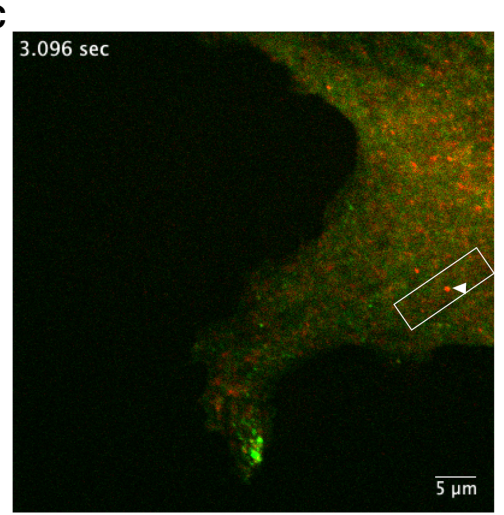

D

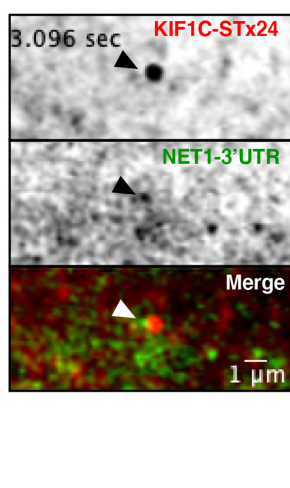

$\mathbf{F}$

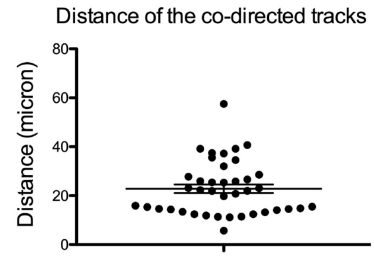

G Mean speed of co-directed tracks

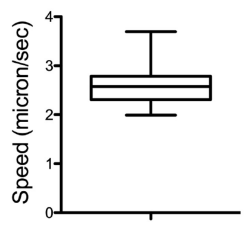

B

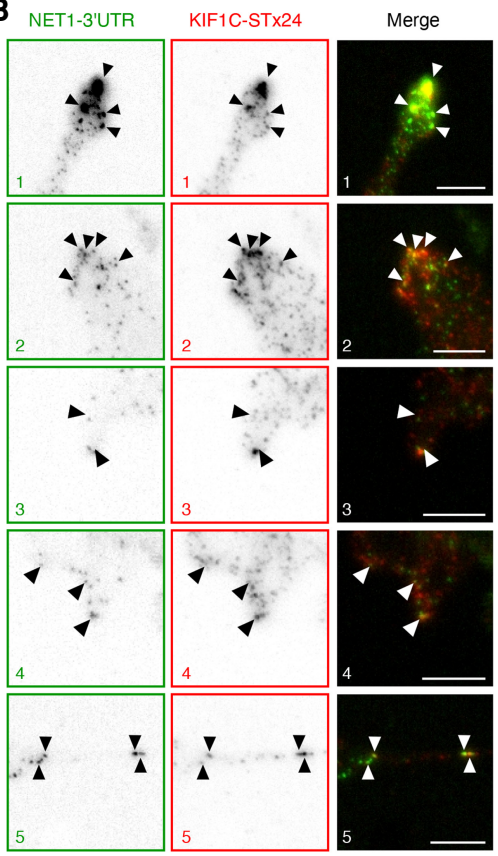

E

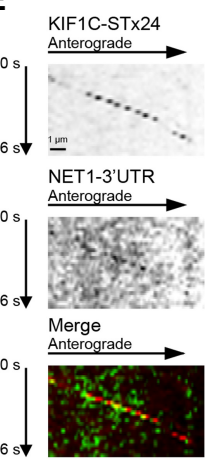

FIGURE 6. The KIF1C motor transports mRNAs containing the Net1 3'UTR to cell protrusions. (A) Images are micrographs of fixed NIH/3T3 cells expressing the $324 \mathrm{bs} /$ Net1 reporter mRNA, MCP-GFP (green), KIF1C-ST 24 protein, and scFv-mScarletl (red). Single molecules of the $\beta 24 \mathrm{bs} /$ Net1 reporter mRNA are visible in green, while single molecules of KIF1C-ST $\times 24$ protein are red. The numbered white boxes are magnified in B. Blue: DNA stained with DAPI. Scale bar is 5 microns. (B) Insets represent magnifications of the boxed areas from the image shown in A. (Left) MCP-GFP signals labeling $324 \mathrm{bs} /$ Net1 mRNAs; (middle) scFv-mScarletl labeling KIF1C-ST 24 protein; (right) merge with mRNAs in green and KIF1C-ST $x_{24}$ in red. Black and white arrowheads indicate colocalization of single molecules of $\beta 24 \mathrm{bs} / \mathrm{Net} 1 \mathrm{mRNAs}$ and KIF1C-ST $\mathrm{m}_{24}$. Scale bar is 5 microns. (C) Snapshot of a live NIH/3T3 cell expressing $\beta 24 b s / N e t 1$ mRNA, MCP-GFP (green), KIF1C-ST $\times 24$ protein, and scFv$\mathrm{mScarletl}(\mathrm{red})$. Snapshot is extracted from Supplemental Movie S9. The white arrowhead indicates a cotransport event of a single molecule of $\beta 24 \mathrm{bs} /$ Net1 mRNA (green) with a KIF1C-ST 24 protein (red). The boxed area is magnified in panels $D$ and $E$. Scale bar is 5 microns. (D)

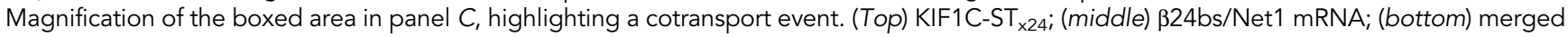
panel with the $324 \mathrm{bs} /$ Net1 mRNA in green and KIF1C-ST 24 in red. Scale bar is 1 micron. (E) Kymograph from Supplemental Movie S9, showing the trajectory of a single molecule of KIF1C-ST ${ }_{x 24}$ (top panel), a single molecule of $\beta 24 \mathrm{bs} /$ Net1 mRNA (middle panel), and the merge (bottom panel). The cellular area shown corresponds to panel D. (F) The graph depicts the distance traveled by cotransported molecules of KIF1C$\mathrm{ST}_{\mathrm{x} 24}$ and $\beta 24 \mathrm{bs} /$ Net1 mRNA. Each data point is a track (40 tracks in total), and the mean and $95 \%$ confidence intervals are shown by horizontal lines. Source data are provided in Supplemental Table S4. (G) Boxplot depicting the mean speed of cotransported molecules of KIF1C-ST 24 and $\beta 24 \mathrm{bs} / \mathrm{Net1} \mathrm{mRNA}$ in NIH/3T3 cells. Speed is microns/second. The vertical bars display the first and last quartile, the box corresponds to the second and third quartiles, and the horizontal line to the mean (40 tracks in total). Source data are provided in Supplemental Table S4. 
to cytoplasmic protrusions of many cell types, where they are anchored at the plus-end of detyrosinated microtubules by APC. Here, we show that these mRNAs associate with the microtubule motor KIF1C and interestingly, they include the KIF1C mRNA itself. We show that APC-dependent mRNAs and KIF1C protein colocalize in protrusions and can also be seen cotransported together along directed tracks. Moreover, the peripheral localization of these mRNAs as well as their microtubule-dependent motion depend on KIF1C, demonstrating that it is an essential motor that transports APC-dependent mRNAs to protrusions. Our data provide a striking in vivo visualization of the cotransport of individual RNA molecules with a specific molecular motor, involved in a widespread RNA transport pathway.

\section{The kinesin KIF1C controls a widespread mammalian mRNA transport system}

RNA localization controls spatial and temporal aspects of gene expression in a variety of species and cell types. Although its significance is better understood in specialized cells such as neurons, recent reports highlight its widespread prevalence, including in cells with a mesenchymal phenotype (Mardakheh et al. 2015; Wang et al. 2017; Fazal et al. 2019; Chouaib et al. 2020; Costa et al. 2020). Our current understanding of the transport mechanisms includes the requirement of cis-acting sequence elements and trans-acting factors, which work with the actin or microtubule networks and motor proteins to bring mRNAs to their destination (Medioni et al. 2012; Cody et al. 2013; Bovaird et al. 2018). Nevertheless, our knowledge of common RNA transport mechanisms that operate in most, if not all cells, is limited. In the case of protrusion mRNAs, which localize in all cell types examined so far, their localization was shown to require APC and detyrosinated microtubules (Mili et al. 2008; Wang et al. 2017). It is not yet known whether APC is transported together with the KIF1C-RNA complexes or whether it is independently transported and subsequently associated with RNAs at the periphery. In vitro studies suggest that motile complexes can be formed between mRNA, APC and KIF3A (Baumann et al. 2020). Our data, however, clearly show the involvement of KIF1C in transporting protrusion mRNAs in vivo. Moreover, APC was shown to accumulate at the leading edge of migrating cells using kinesin-1 and kinesin-2 (Mimori-Kiyosue et al. 2000; Nakamura et al. 2001; Ruane et al. 2016). The use of distinct motors suggests an independent transport for APC and protrusion mRNAs. It is also possible that this diversity of motors reflects differences between cell types, as has been described for the $\beta$-actin RNA that uses different motors in neurons and fibroblasts (see Introduction; Latham et al. 2001; Ma et al. 2011; Nalavadi et al. 2012; Song et al. 2015). One potential reason for using different motors could pertain to the dynamics and transport speeds required in each case. In this context, the fact that KIF1C appears to be the fastest human cargo transporter (Lipka et al. 2016) might provide an advantage that could underlie its preferential use in the highly dynamic mesenchymal cells. Furthermore, kinesins other than KIF1C, such as KIF5B, contribute to localization of APC-dependent RNAs (Yasuda et al. 2017). We speculate that they may function in specialized cells or affect different aspects of localization that are distinct from transport per se. In line with this idea, protrusion mRNAs display a complex translational regulation concomitant to the protrusion dynamics and a local reorganization of RNA clusters (Moissoglu et al. 2019), likely requiring a number of yet uncharacterized actors.

Another important question deals with the adaptors that link KIF1C to mRNAs. On one hand, proteomic data showed that KIF1C protein interacts with the exon junction complex (EJC; Hein et al. 2015). The EJC is assembled on spliced RNAs and serves as an interaction platform for proteins that direct mRNA export, localization, translation and nonsense-mediated mRNA decay (NMD), suggesting a role for the EJC in the transport of protrusion mRNAs. On the other hand, regions rich in $G$ and $A$ nucleotides are present in the 3'UTR of APC-dependent RNAs and are part of the localization element (Costa et al. 2020; Moissoglu et al. 2020). Furthermore, the $3^{\prime}$ UTR is sufficient to direct protrusion localization of intronless exogenous constructs (Moissoglu et al. 2019, 2020), indicating that a potential KIF1C recruitment through exon-exon junctions might not be necessary for protrusion localization. Specific RNA-binding proteins could serve as adaptors that mediate KIF1C recruitment similar to the model suggested for other RNA transport complexes. However, given that high-throughput mRNA-protein cross-linking approaches previously showed that KIF1C directly binds mRNAs (Baltz et al. 2012; Castello et al. 2012), an alternative interesting possibility would be that in this case the motor directly selects and binds to its RNA cargo.

\section{KIF1C triggers mRNA clustering in cytoplasmic protrusions}

Our live imaging experiments show that reporter mRNAs are transported predominantly as single molecules during the early stages of cell spreading, reminiscing the singlemolecule appearance of RAB13 mRNA in actively protruding regions in migrating cells (Moissoglu et al. 2019). Remarkably, APC-dependent mRNAs coalesce into higher order clusters at peripheral regions during later time points of cell spreading, and this phenotype depends specifically on KIF1C. We think that clustering is not merely a consequence of peripheral mRNA accumulation, because we have not observed it in the absence of KIF1C even at protrusions still containing substantial numbers of single mRNA molecules. We rather favor the explanation that clustering is a separate function of KIF1C that is temporally 
and spatially regulated. Given that these clusters contain stably anchored mRNAs (Mili et al. 2008), we envision that KIF1C switches from a microtubule motor to an mRNA anchoring module promoting clustering. A similar switch has been observed in Drosophila oocytes, whereby Dynein converts from a motor of gurken mRNA to a static anchor at its final destination (Delanoue et al. 2007). Such a switch on KIF1C may take place on pre-existing motor molecules as they reach the periphery or may be a function of newly synthesized KIF1C translated from its peripherally localized mRNA. It is still unclear how such a switch would occur and/or whether it might additionally involve a change in the RNA-binding mode of KIF1C (direct or indirect through other RNA-binding proteins). Clusters of APC-dependent mRNAs have been previously reported to be heterogeneous and to contain translationally silent mRNAs (Moissoglu et al. 2019). Thus, overall our results point to a spatially and temporally controlled mRNA clustering role of KIF1C that is separate from its motor function and that might be coordinated with translational regulation.

\section{RNA transported by KIF1C mediates diverse functions at cell protrusions}

Peripheral localization of APC-dependent RNAs promotes cell migration (Wang et al. 2017). Specifically, approaches targeting the localization elements of these mRNAs, as a group (Wang et al. 2017) or individually (Moissoglu et al. 2020), resulted in inhibition of cell migration. These effects are likely due to a requirement for locally translating these mRNAs for full activation of the encoded proteins (Moissoglu et al. 2020). Interestingly, KIF1C has been shown to control adhesion dynamics and cell migration (Theisen et al. 2012). It was proposed to act via the trafficking of $\alpha 5 \beta 1$ integrins. While we cannot completely rule out that KIF1C indirectly affects peripheral RNA localization through altering adhesion dynamics, we consider this highly unlikely given the physical interaction and cotransport of KIF1C and RNAs that we report here. Instead, our results indicate that the transport of APC-dependent mRNAs to the periphery by KIF1C likely itself contributes to the mechanism by which this kinesin controls cell migration. Along this line, the GO terms associated with the top 200 KIF1C-associated mRNAs presented in this study (i.e., organelle organization; plasma membrane bounded cell projection organization; microtubule-based transport; cilium organization; Supplemental Table S5) indicate how KIF1C-mediated mRNA transport could impact processes related to cell motility.

\section{KIF1C protein localizes its own mRNAs to cell protrusions: a transport feedback loop?}

The KIF1C transcript localizes to cytoplasmic protrusions in mammalian cells. Moreover, it colocalizes with KIF1C pro- tein in protrusions (Chouaib et al. 2020), and we show here that the KIF1C protein physically associates with its own mRNA. This local accumulation of KIF1C could be involved in an RNA clustering and anchoring mechanism as discussed above, but it could also serve to transport additional mRNAs by alternating back-and-forth movements on the cytoskeleton. Indeed, locally translated KIF1C protein would allow the motor to explore the local cytoplasm and transport back additional mRNAs to protrusions using the same MT tracks. Such a bidirectional motility has been reported for KIF1C and it is mediated by the scaffold protein Hook3. This protein forms a complex between dynein and KIF1C (Kendrick et al. 2019), and regulates their activities to allow the motor to perform multiple transport cycles while avoiding a tug-of-war between opposite motors (Siddiqui et al. 2019). The fact that KIF1C also brings its own mRNA to protrusions suggests the possible existence of a positive feedback loop in which locally translated KIF1C provides additional motor molecules to sustain the persistent and directional transport of its RNA cargoes, to locally maintain protrusive extensions during cell movement.

\section{MATERIALS AND METHODS}

\section{Generation and maintenance of cell lines}

The HeLa-Kyoto cells stably transfected with the KIF1C-GFP BAC were previously described (Poser et al. 2008; Maliga et al. 2013; Chouaib et al. 2020). HeLa Flp-in H9 (a kind gift of S. Emiliani) and the BAC-GFP cells were maintained in Dulbecco's Modified Eagle Medium (DMEM, Gibco) supplemented with 10\% fetal bovine serum (FBS, Sigma), $100 \mathrm{U} / \mathrm{mL}$ penicillin/streptomycin (Sigma) and with $400 \mu \mathrm{g} / \mathrm{mL}$ G418 (Gibco) for the HeLa-Kyoto KIF1C-GFP tagged BAC cells. NIH/3T3 cells were maintained in DMEM supplemented with $10 \%$ calf serum, sodium pyruvate and penicillin/streptomycin at $37^{\circ} \mathrm{C}, 5 \% \mathrm{CO}_{2}$. MDA-MB-231 human breast cancer cells (ATCC) were grown in Leibovitz's L15 media supplemented with $10 \%$ fetal bovine serum and penicillin/ streptomycin at $37^{\circ} \mathrm{C}$ in atmospheric air. Stable HeLa cell lines expressing a KIF1C-GFP cDNA were created using the Flp-in system in HeLa H9 cells. Flp-in integrants were selected on hygromycin $\left(150 \mu \mathrm{g} \mathrm{mL}^{-1}\right)$. To generate cell lines expressing RNA reporters, $\mathrm{NIH} / 3 \mathrm{~T} 3$ cells were infected with lentivirus expressing tdMCPGFP (Addgene plasmid \#40649), and GFP-expressing cells with a low level of GFP expression were sorted by FACS. This stable population was infected with plnducer20-based reporter constructs expressing $\beta$-globin followed by $24 x M S 2$ binding sites and the mouse Net1, Rab13, or control 3'UTRs (pIND20- $324 b s$ /

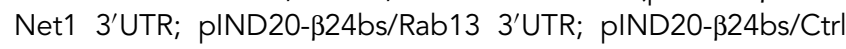
3'UTR; Moissoglu et al, 2019). Stable lines were selected with geneticin (Thermo Fisher Scientific) and expression of the reporter was induced by addition of $1 \mu \mathrm{g} / \mathrm{mL}$ doxycycline (Fisher Scientific) 2-3 h before imaging.

For the two-color tracking experiment, NIH/3T3 cells expressing the pIND20- $\beta 24 \mathrm{bs} /$ Net1 reporter and tdMCP-GFP described below were modified as follows. Stable expression of scFvmScarletl-GB1 in NIH/3T3 cells was achieved by lentiviral- 
mediated integration of a plasmid derived from Addgene (\#60906) and sorted by FACS with a low level of mScarletl expression. Then a lentivirus expressing a KIF1C fusion with 24 repeats of the GCN4 peptide array was used to infect these NIH/3T3 cells allowing the detection of KIF1C-STX24 protein with ScFvmScarletl-GB1.

\section{Treatments with siRNAs and drugs}

HeLa cells were seeded on $0.17 \mathrm{~mm}$ glass coverslips deposited in six-well plates. Cells were transfected at $70 \%$ confluency using JetPrime (Polyplus). Double-stranded siRNAs (30 pmoles) were diluted into $200 \mu \mathrm{L}$ of JetPrime buffer. JetPrime reagent was added $(4 \mu \mathrm{L})$ and the mixture was vortexed. After $10 \mathrm{~min}$ at room temperature (RT), it was added to the cells grown in $2 \mathrm{~mL}$ of serum-containing medium. After $24 \mathrm{~h}$, the transfection medium was replaced with fresh growth medium and cells were fixed $24 \mathrm{~h}$ later. The sequences of the siRNA were: KIF1C: 5'-CCCAUGCCGUCUUUACCAUdCdG; control: 5'-CAACAGAAGGAGAGCGAAAdTdT. For knockdown of human or mouse Kif1c the following additional siRNAs were used: Hs_KIF1C_5 (Qiagen cat\# SI02655401), Hs_KIF1C_6 (Qiagen cat\# SI02781331), Hs_KIF1C_7 (Qiagen cat\# SI02781338), Hs_KIF1C_8 (Qiagen cat\# SI03019744), Mm_Kif1c_2 (Qiagen cat\# SI00239687), Mm_Kif1c_3 siRNA (Qiagen cat\# SI00239694), and AllStars negative control siRNA (Qiagen cat\# 1027281). siRNAs were delivered into cells using Lipofectamine RNAiMAX (Thermo Fisher Scientific, cat\# 13778-150) according to the manufacturer's instructions. Cells were assayed $72 \mathrm{~h}$ after siRNA transfection.

For drug treatments, $10 \mu \mathrm{M}$ nocodazole or Cytochalasin $\mathrm{D}$, or an equal volume of DMSO, were added to the growth media for $15 \mathrm{~min}$.

\section{Plasmid construction}

Plasmids were generated with standard molecular biology techniques. The GFP-APC plasmid was a gift from Inke Nathke. The KIF1C-mCherry plasmid was a gift from Anne Straube (Addgene plasmid \#130978). The inducible constructs expressing MS2-containing RNA reporters (pIND20- $\beta 24 b s / N e t 1 \quad 3$ 'UTR; pIND20- $\beta 24 \mathrm{bs} /$ Rab13 3'UTR) are described in Moissoglu et al. (2019). They contain the human $\beta$-globin gene followed by $24 \times$ MS2 binding sites and the mouse Net1, Rab13, or control 3'UTRs. The control reporter (pIND20- $\beta 24 \mathrm{bs} / \mathrm{Ctrl} 3^{\prime} \mathrm{UTR}$ ) carries a short, random, vector-derived UTR sequence. To generate the KIF1C-SunTagx24 cell lines, the KIF1C cDNA was amplified by PCR and cloned in pHRdSV40-K560-GCN4 $\times 24$ (Addgene \#72229), in place of the K560 cDNA and upstream of the SunTag. Then the KIF1C-STx24 sequence was PCR amplified and cloned into pHAGE-Ubc-MCP-YFP (Addgene \#31230) in place of the MCP-GFP sequence. Maps and sequences are available upon request.

\section{Immunoprecipitation and microarrays}

HeLa cells containing the KIF1C-GFP BAC were grown to near confluence in $10 \mathrm{~cm}$ plates, and two plates were used per IP. Cells were rinsed in ice-cold PBS, and all subsequent manipulations were performed at $4^{\circ} \mathrm{C}$. Cells were scraped in HTNG buffer
(20 mM HEPES-KOH pH 7.9, 150 mM NaCl, 1\% Triton X-100, 10\% glycerol, $1 \mathrm{mM} \mathrm{MgCl} 2,1 \mathrm{mM}$ EGTA), containing an antiprotease cocktail (Roche Diagnostic). Cells were incubated for 20 min on a rotating wheel, and cellular debris were removed by centrifugating the extracts $10 \mathrm{~min}$ at 20,000 g. Beads coated with GFP-trap antibody (ChromoTek), or uncoated as control, were washed in HNTG ( $25 \mu \mathrm{L}$ of beads per IP). Beads were incubated $1 \mathrm{~h}$ with a control extract to saturate nonspecific binding and then incubated with the proper extract. After $4 \mathrm{~h}$ of incubation on a rotating wheel, beads were washed four times in HNGT with anti-protease, and twice with PBS. Beads were then incubated with TRIzol to extract RNAs, and RNA purification was done as recommended by the manufacturer. The resulting RNAs were amplified and converted into cDNAs by the WT PICO kit (Thermo Fisher), and hybridized on an HTA 2.0 chip on an Affymetrix platform (Thermo Fisher). Experiments were performed in duplicates, data were normalized and averaged. Data are deposited on GEO with the following accession number: GSE161316.

For GFP-APC immunoprecipitation, cells were lysed with a buffer containing $50 \mathrm{mM}$ Tris $\mathrm{pH} 7.4,1 \%$ Triton X-100, $75 \mathrm{mM} \mathrm{NaCl}$, $10 \mathrm{mM} \mathrm{MgCl} 2,10 \%$ glycerol and a cocktail of protease and phosphatase inhibitors (Thermo Fischer Scientific, cat\# 1861281). Lysates were cleared by centrifugation and mixed with GFPTrap Magnetic Agarose beads (Chromotek, cat\# gtma-10) for $1.5 \mathrm{~h}$, at $4^{\circ} \mathrm{C}$. Immobilized complexes were eluted with Laemmli's buffer and analyzed by SDS-PAGE and immunoblotting.

\section{Western blot}

For western blot detection the following antibodies were used: anti-GFP rabbit polyclonal (Invitrogen, cat\# A11122, 1/2000 dilution), anti-KIF1C rabbit polyclonal (Proteintech, cat\# 12760-1-AP), anti-KIF1C rabbit polyclonal (Bethyl Laboratories, cat\# A301070A, 1/2000 dilution), anti- $\alpha$-tubulin mouse monoclonal (Sigma-Aldrich, cat\# T6199, 1/10,000 dilution), anti-mCherry mouse monoclonal [1C51] (Abcam, cat\# ab125096, 1/2000 dilution).

\section{RNA analyses}

For total RNA analysis, cells were lysed with TRIzol LS reagent (Thermo Fisher Scientific, cat\# 10296010) and RNA was extracted according to the manufacturer's instructions. Isolated RNA was treated with RQ1 RNase-free DNase (M6101, Promega) and analyzed with the nCounter system (NanoString Technologies) using a custom-made codeset. Data were processed using nSolver analysis software (NanoString technologies).

\section{Single-molecule FISH}

Cells grown on glass coverslips were fixed for $20 \mathrm{~min}$ at RT with $4 \%$ paraformaldehyde diluted in PBS, and permeabilized with $70 \%$ ethanol overnight at $4{ }^{\circ} \mathrm{C}$. For smFISH, we used a set of 44 amino-modified oligonucleotide probes against the GFP-IRESNeo sequence (sequences given in Supplemental Table S2). Each oligonucleotide probe contained 4 primary amines that were conjugated to Cy3 using the Mono-Reactive Dye Pack 
(PA23001, GE Healthcare Life Sciences). To this end, the oligos were precipitated with ethanol and resuspended in water. For labeling, $4 \mu \mathrm{g}$ of each probe was incubated with $6 \mu \mathrm{L}$ of Cy3 $(1 / 5$ of a vial resuspended in $30 \mu \mathrm{L}$ of DMSO), and $14 \mu \mathrm{L}$ of carbonate buffer $0.1 \mathrm{M} \mathrm{pH} 8.8$, overnight at RT and in the dark, after extensive vortexing. The next day, $10 \mu \mathrm{g}$ of yeast tRNAs were added and the probes were precipitated several times with ethanol until the supernatant lost its pink color. For hybridization, fixed cells were washed with PBS and hybridization buffer (15\% formamide in $1 \times S S C)$, and then incubated overnight at $37^{\circ} \mathrm{C}$ in the hybridization buffer also containing $130 \mathrm{ng}$ of the probe set for $100 \mu \mathrm{L}$ of final volume, $0.34 \mathrm{mg} / \mathrm{mL}$ tRNA (Sigma), $2 \mathrm{mM} \mathrm{VRC} \mathrm{(Sigma),} 0.2$ $\mathrm{mg} / \mathrm{mL}$ RNase-free BSA (Roche Diagnostic), and 10\% Dextran sulfate. The next day, the samples were washed twice for $30 \mathrm{~min}$ in the hybridization buffer at $37^{\circ} \mathrm{C}$, and rinsed in PBS. Coverslips were then mounted using Vectashield containing DAPI (Vector laboratories, Inc.).

For smiFISH (Tsanov et al. 2016), 24 to 48 unlabeled primary probes were used (sequences given in Supplemental Table S2). In addition to hybridizing to their targets, these probes contained a FLAP sequence that was hybridized to a secondary fluorescent oligonucleotide. To this end, 40 pmoles of primary probes were prehybridized to 50 pmoles of secondary probe in $10 \mu \mathrm{L}$ of 100 $\mathrm{mM} \mathrm{NaCl}, 50 \mathrm{mM}$ Tris- $\mathrm{HCl}, 10 \mathrm{mM} \mathrm{MgCl}$, $\mathrm{pH}$ 7.9. Hybridization was performed at $85^{\circ} \mathrm{C}$ for $3 \mathrm{~min}, 65^{\circ} \mathrm{C}$ for $3 \mathrm{~min}$, and $25^{\circ} \mathrm{C}$ for $5 \mathrm{~min}$. The final hybridization mixture contained the probe duplexes ( $2 \mu \mathrm{L}$ per $100 \mu \mathrm{L}$ of final volume), with $1 \times$ SSC, $0.34 \mathrm{mg} / \mathrm{mL}$ tRNA (Sigma), 15\% Formamide, $2 \mathrm{mM}$ VRC (Sigma), $0.2 \mathrm{mg} / \mathrm{mL}$ RNase-free BSA, 10\% Dextran sulfate. Slides were then processed as above. For Supplemental Figure S1A, the probes used were RNA and not DNA (sequence in Supplemental Table S2). The protocol was similar except that hybridization was performed at $48^{\circ} \mathrm{C}$ and that $50 \mathrm{ng}$ of the primary probe (total amount of the pool of probes) and $30 \mathrm{ng}$ of the secondary probes were used per $100 \mu \mathrm{L}$ of hybridization mix.

For FISH of mouse cells, cells plated on fibronectin-coated coverslips were fixed for 20 min at RT with $4 \%$ paraformaldehyde in PBS. FISH was performed with the ViewRNA ISH Cell Assay kit (Thermo Fisher Scientific) according to the manufacturer's instructions. The flowing probe sets were used: Kif1c (cat\# VB63200442), Net1 (cat\# VB1-3034209), Rab13 (cat\# VB1-14374), Ddr2 (cat\# VB1-14375), Dynll2 (cat\# VB1-18646), Cyb5r3 (cat\# VB1-18647). To detect polyA+ RNAs, LNA modified oligodT probes (30 nt) labeled with ATTO 655 were added during hybridization, preamplification, amplification, and last hybridization steps of the ViewRNA ISH Cell Assay. Cell mask stain (Thermo Fisher Scientific) was used to identify the cell outlines. Samples were mounted with ProLong Gold antifade reagent (Thermo Scientific)

\section{Imaging of fixed cells}

Microscopy slides were imaged on a Zeiss Axioimager Z1 widefield microscope equipped with a motorized stage, a camera scMOS ZYLA $4.2 \mathrm{MP}$, using a $63 \times$ or $100 \times$ objective (Plan Apochromat; $1.4 \mathrm{NA}$; oil). Images were taken as z-stacks with one plane every $0.3 \mu \mathrm{m}$. The microscope was controlled by MetaMorph and figures were constructed using ImageJ, Adobe Photoshop and Illustrator. For the small smiFISH screen, 96-well plates were imaged on an Opera Phenix High-Content Screening System (PerkinElmer), with a $63 \times$ water-immersion objective (NA 1.15). Three-dimensional images were acquired, consisting of 35 slices with a spacing of $0.3 \mu \mathrm{m}$. FISH images of mouse cells were obtained using a Leica SP8 confocal microscope, equipped with a HC PL APO $63 \times$ CS2 objective. Z-stacks through the cell volume were obtained and maximum intensity projections were used for subsequent analysis.

\section{Image analysis and quantifications}

Automated nuclear and cell segmentation was performed with a custom algorithm based on the U-net deep convolutional network (Ronneberger et al. 2015). Nuclear segmentation was performed with the DAPI channel; cell segmentation was performed with the autofluorescence of the actual smFISH image. For segmentation, 3D images were projected into 2D images as described previously (Tsanov et al. 2016). Messenger RNAs were detected with FISH-quant (Mueller et al. 2013) by applying a local maximum detection on LoG filtered images. For calculation of Peripheral Distribution Index (PDI) a custom Matlab script was used. The code is described and is available in Stueland et al. (2019).

\section{Imaging of live cells}

Live imaging (for dual visualization of $\beta 24 \mathrm{bs} / \mathrm{Net} 1$ reporter RNA and KIF1C-ST x24 $_{2}$ protein) was done using a spinning disk confocal microscope (Nikon Ti with a Yokogawa CSU-X1 head) operated by the Andor iQ3 software. Acquisitions were performed using a $100 \times$ objective (CF1 PlanApo $\lambda 1.45$ NA oil), and an EMCCD iXon897 camera (Andor). For two-color imaging, samples were sequentially excited at 488 and $540 \mathrm{~nm}$. We imaged at a rate of $7.36 \mathrm{fps}$ for $52 \mathrm{sec}$. The power of illuminating light and the exposure time were set to the lowest values that still allowed visualization of the signal. This minimized bleaching, toxicity and maximized the number of frames that were collected. Cells were maintained in anti-bleaching live cell visualization medium (DMEM $^{\text {gfp}}$; Evrogen), supplemented with $10 \%$ fetal bovine serum at $37^{\circ} \mathrm{C}$ in $5 \% \mathrm{CO}_{2}$ and rutin at a final concentration of $20 \mathrm{mg} / \mathrm{L}$.

Live imaging (for $\beta 24 \mathrm{bs} /$ Net1 reporter RNA tracking) was done using a Nikon Eclipse Ti2-E inverted microscope, equipped with a motorized stage, a Yokogawa CSU-X1 spinning disk confocal scanner unit, and operated using NIS-elements software. Acquisitions were performed using an Apochromat TIRF 100x oil immersion objective (N.A. 1.49, W.D. $0.12 \mathrm{~mm}$, F.O.V. 22 $\mathrm{mm}$ ) and a Photometrics Prime 95B Back-illuminated sCMOS camera with $\mathrm{W}$-view Gemini Image splitter. Constant $37^{\circ} \mathrm{C}$ temperature and $5 \% \mathrm{CO}_{2}$ were maintained using a Tokai Hit incubation system. Cells were plated on fibronectin $(2 \mathrm{mg} / \mathrm{mL})$-coated 35 $\mathrm{mm}$ glass bottom dishes, and samples were excited using a 488 $\mathrm{nm}(20 \mathrm{mw})$ laser line and imaged at a rate of $6.66 \mathrm{fps}$ for $60 \mathrm{sec}$.

\section{Live cell imaging quantification}

Images were processed for brightness/contrast, cropped and annotated using ImageJ/FIJI. Kymographs were generated using standard ImageJ/Fiji plugins. Film presentation in figures and 
videos were edited in ImageJ/Fiji. Bicolor tracking of KIF1CSTx24 proteins and $\beta 24 \mathrm{bs} /$ Net1 mRNAs was performed using the Manual Tracking plugin in ImageJ/Fiji.

Single color tracking of $\beta 24 \mathrm{bs} /$ Net1 mRNAs was performed using TrackMate plugin in ImageJ/Fiji. For every cell, all tracks lasting for $>2.5 \mathrm{sec}$ (approximately 17 consecutive frames) were used for analysis. Values of "Track displacement," "Linearity of forward progression," "track duration," and "mean speed" were extracted and plotted. "Track displacement" is defined as the distance from the first to the last spot of the track. "Linearity of forward progression" is the mean straight line speed divided by the mean speed; where mean straight line speed is defined as the net displacement divided by the total track time. MSD and velocity autocorrelation of tracks was determined using MSDanalyzer (https://github.com/tinevez/msdanalyzer). For RNA cluster analysis, TrackMate was used to identify spots and extract intensity values. Frequency histograms of spot intensities were plotted using GraphPad Prism software.

\section{SUPPLEMENTAL MATERIAL}

Supplemental material is available for this article.

\section{ACKNOWLEDGMENTS}

We thank the staff of the MRI imaging facility for their technical support. This project was supported by France Biolmaging (ANR-10-INBS-04), the Agence Nationale de la Recherche (ANR11-BSV8-018-02, ANR-14-CE10-0018-01 and ANR-19-CE120007-03), and the Ligue Nationale Contre le Cancer and the Fondation pour la Recherche Médicale. This work was supported by the Labex EpiGenMed, from the framework "Investissements d'avenir." This work was supported in part by the Intramural Research Program of the National Cancer Institute, National Institutes of Health.

Author contributions: E.B. conceived the study with S.M. and K. Z. Experiments were performed by E.B., X.P., K.M., E.C., Ti.W., M. P., M.C.R., and R.C. A.I., K.M., S.M., Th.W., and F.M. analyzed images. E.B., X.P., K.M., S.M., E.C., Th.W., M.P., A.I., F.M., M.C.R., and R.C. analyzed the data. E.B., X.P., and K.M. prepared the figures. E.B., X.P., K.M., and S.M. wrote the manuscript.

Received December 7, 2020; accepted September 1, 2021

\section{REFERENCES}

Bahmanyar S, Nelson WJ, Barth AIM. 2009. Role of APC and its binding partners in regulating microtubules in mitosis. Adv Exp Med Biol 656: 65-74. doi:10.1007/978-1-4419-1145-2_6

Baltz AG, Munschauer M, Schwanhäusser B, Vasile A, Murakawa $Y$, Schueler M, Youngs N, Penfold-Brown D, Drew K, Milek M, et al. 2012. The mRNA-bound proteome and its global occupancy profile on protein-coding transcripts. Mol Cell 46: 674-690. doi:10 .1016/j.molcel.2012.05.021

Barth AIM, Caro-Gonzalez HY, Nelson WJ. 2008. Role of adenomatous polyposis coli (APC) and microtubules in directional cell migration and neuronal polarization. Semin Cell Dev Biol 19: 245251. doi:10.1016/j.semcdb.2008.02.003

Baumann S, Komissarov A, Gili M, Ruprecht V, Wieser S, Maurer SP. 2020. A reconstituted mammalian APC-kinesin complex selective- ly transports defined packages of axonal mRNAs. Sci Adv 6: eaaz1588. doi:10.1126/sciadv.aaz1588

Berleth T, Burri M, Thoma G, Bopp D, Richstein S, Frigerio G, Noll M, Nüsslein-Volhard C. 1988. The role of localization of bicoid RNA in organizing the anterior pattern of the Drosophila embryo. EMBO J 7: 1749-1756. doi:10.1002/j.1460-2075.1988.tb03004.x

Bovaird S, Patel D, Padilla J-CA, Lécuyer E. 2018. Biological functions, regulatory mechanisms, and disease relevance of RNA localization pathways. FEBS Lett 592: 2948-2972. doi:10.1002/1873-3468 .13228

Cai D, McEwen DP, Martens JR, Meyhofer E, Verhey KJ. 2009. Single molecule imaging reveals differences in microtubule track selection between Kinesin motors. PLoS Biol 7: e1000216. doi:10 .1371 /journal.pbio. 1000216

Carson JH, Worboys K, Ainger K, Barbarese E. 1997. Translocation of myelin basic protein mRNA in oligodendrocytes requires microtubules and kinesin. Cell Motil Cytoskeleton 38 318-328. doi:10 .1002/(SICl)1097-0169(1997)38:4<318::AID-CM2>3.0.CO;2-\#

Castello A, Fischer B, Eichelbaum K, Horos R, Beckmann BM, Strein C, Davey NE, Humphreys DT, Preiss T, Steinmetz LM, et al. 2012. Insights into RNA biology from an atlas of mammalian mRNAbinding proteins. Cell 149: 1393-1406. doi:10.1016/j.cell.2012 .04 .031

Chicurel ME, Singer RH, Meyer CJ, Ingber DE. 1998. Integrin binding and mechanical tension induce movement of mRNA and ribosomes to focal adhesions. Nature 392: 730-733. doi:10.1038/ 33719

Chin A, Lécuyer E. 2017. RNA localization: making its way to the center stage. Biochim Biophys Acta 1861: 2956-2970. doi:10.1016/j .bbagen.2017.06.011

Chouaib R, Safieddine A, Pichon X, Imbert A, Kwon OS, Samacoits A, Traboulsi A-M, Robert M-C, Tsanov N, Coleno E, et al. 2020. A dual protein-mRNA localization screen reveals compartmentalized translation and widespread co-translational RNA targeting. Dev Cell 54: 773-791. doi:10.1016/j.devcel.2020.07.010

Chrisafis G, Wang T, Moissoglu K, Gasparski AN, Ng Y, Weigert R, Lockett SJ, Mili S. 2020. Collective cancer cell invasion requires RNA accumulation at the invasive front. Proc Natl Acad Sci 117: 27423-27434. doi:10.1073/pnas.2010872117

Cody NAL, lampietro C, Lécuyer E. 2013. The many functions of mRNA localization during normal development and disease: from pillar to post. WIREs Dev Biol 2: 781-796. doi:10.1002/ wdev.113

Condeelis J, Singer RH. 2005. How and why does $\beta$-actin mRNA target? Biol Cell 97: 97-110. doi:10.1042/BC20040063

Costa G, Bradbury JJ, Tarannum N, Herbert SP. 2020. RAB13 mRNA compartmentalisation spatially orients tissue morphogenesis. EMBO J 39: e106003. doi:10.15252/embj.2020106003

Delanoue R, Herpers B, Soetaert J, Davis I, Rabouille C. 2007. Drosophila Squid/hnRNP helps Dynein switch from a gurken mRNA transport motor to an ultrastructural static anchor in sponge bodies. Dev Cell 13: 523-538. doi:10.1016/j.devcel.2007.08.022

Dunn S, Morrison EE, Liverpool TB, Molina-París C, Cross RA, Alonso MC, Peckham M. 2008. Differential trafficking of Kif5c on tyrosinated and detyrosinated microtubules in live cells. J Cell Sci 121: 1085-1095. doi:10.1242/jcs.026492

Eliscovich C, Singer RH. 2017. RNP transport in cell biology: the long and winding road. Curr Opin Cell Biol 45: 38-46. doi:10.1016/j ceb.2017.02.008

Falley K, Schütt J, Iglauer P, Menke K, Maas C, Kneussel M, Kindler S, Wouters FS, Richter D, Kreienkamp H-J. 2009. Shank1 mRNA: dendritic transport by kinesin and translational control by the 5'untranslated region. Traffic 10: 844-857. doi:10.1111/j.16000854.2009.00912.x 
Fazal FM, Han S, Parker KR, Kaewsapsak P, Xu J, Boettiger AN, Chang HY, Ting AY. 2019. Atlas of subcellular RNA localization revealed by APEX-seq. Cell 178: 473-490.e26. doi:10.1016/j.cell .2019.05.027

Forrest KM, Gavis ER. 2003. Live imaging of endogenous RNA reveals a diffusion and entrapment mechanism for nanos mRNA localization in Drosophila. Curr Biol 13: 1159-1168. doi:10.1016/S09609822(03)00451-2

Fusco D, Accornero N, Lavoie B, Shenoy SM, Blanchard J-M, Singer RH, Bertrand E. 2003. Single mRNA molecules demonstrate probabilistic movement in living mammalian cells. Curr Biol 13: 161-167. doi:10.1016/S0960-9822(02)01436-7

Gagnon JA, Mowry KL. 2011. Molecular motors: directing traffic during RNA localization. Crit Rev Biochem Mol Biol 46: 229-239. doi:10.3109/10409238.2011.572861

Hein MY, Hubner NC, Poser I, Cox J, Nagaraj N, Toyoda Y, Gak IA, Weisswange I, Mansfeld J, Buchholz F, et al. 2015. A human interactome in three quantitative dimensions organized by stoichiometries and abundances. Cell 163: 712-723. doi:10.1016/j.cell .2015.09.053

Jimbo T, Kawasaki Y, Koyama R, Sato R, Takada S, Haraguchi K, Akiyama T. 2002. Identification of a link between the tumour suppressor APC and the kinesin superfamily. Nat Cell Biol 4: 323-327. doi:10.1038/ncb779

Kanai Y, Dohmae N, Hirokawa N. 2004. Kinesin transports RNA: isolation and characterization of an RNA-transporting granule. Neuron 43: 513-525. doi:10.1016/j.neuron.2004.07.022

Katz ZB, Wells AL, Park HY, Wu B, Shenoy SM, Singer RH. 2012. $\beta$ Actin mRNA compartmentalization enhances focal adhesion stability and directs cell migration. Genes Dev 26: 1885-1890. doi:10.1101/gad.190413.112

Kendrick AA, Dickey AM, Redwine WB, Tran PT, Vaites LP, Dzieciatkowska M, Harper JW, Reck-Peterson SL. 2019. Hook3 is a scaffold for the opposite-polarity microtubule-based motors cytoplasmic dynein-1 and KIF1C. J Cell Biol 218: 2982-3001. doi:10 .1083/jcb.201812170

Kislauskis EH, Li Z, Singer RH, Taneja KL. 1993. Isoform-specific 3'-untranslated sequences sort $\alpha$-cardiac and $\beta$-cytoplasmic actin messenger RNAs to different cytoplasmic compartments. J Cell Biol 123: $165-172$. doi:10.1083/jcb.123.1.165

Latham VM, Yu EHS, Tullio AN, Adelstein RS, Singer RH. 2001. A Rhodependent signaling pathway operating through myosin localizes $\beta$-actin mRNA in fibroblasts. Curr Biol 11: 1010-1016. doi:10 .1016/S0960-9822(01)00291-3

Liao G, Mingle L, Van De Water L, Liu G. 2015. Control of cell migration through mRNA localization and local translation. Wiley Interdiscip Rev RNA 6: 1-15. doi:10.1002/wrna.1265

Lin AC, Holt CE. 2007. Local translation and directional steering in axons. EMBO J 26: 3729-3736. doi:10.1038/sj.emboj.7601808

Lipka J, Kapitein LC, Jaworski J, Hoogenraad CC. 2016. Microtubulebinding protein doublecortin-like kinase 1 (DCLK1) guides kinesin3-mediated cargo transport to dendrites. EMBO J 35: 302-318. doi:10.15252/embj.201592929

Ma B, Savas JN, Yu M-S, Culver BP, Chao MV, Tanese N. 2011. Huntingtin mediates dendritic transport of $\beta$-actin mRNA in rat neurons. Sci Rep 1: 140. doi:10.1038/srep00140

Maliga $Z$, Junqueira $M$, Toyoda $Y$, Ettinger A, Mora-Bermúdez $F$, Klemm RW, Vasilj A, Guhr E, Ibarlucea-Benitez I, Poser I, et al. 2013. A genomic toolkit to investigate kinesin and myosin motor function in cells. Nat Cell Biol 15: 325-334. doi:10.1038/ncb2689

Mardakheh FK, Paul A, Kümper S, Sadok A, Paterson H, Mccarthy A, Yuan Y, Marshall CJ. 2015. Global analysis of mRNA, translation, and protein localization: local translation is a key regulator of cell protrusions. Dev Cell 35: 344-357. doi:10.1016/j.devcel.2015.10 .005
Martin KC, Zukin RS. 2006. RNA trafficking and local protein synthesis in dendrites: an overview. J Neurosci 26: 7131-7134. doi:10.1523/ JNEUROSCI.1801-06.2006

Medioni C, Mowry K, Besse F. 2012. Principles and roles of mRNA localization in animal development. Development 139: 3263-3276. doi:10.1242/dev.078626

Mili S, Moissoglu K, Macara IG. 2008. Genome-wide screen identifies localized RNAs anchored at cell protrusions through microtubules and APC. Nature 453: 115-119. doi:10.1038/nature06888

Mimori-Kiyosue Y, Shiina N, Tsukita S. 2000. Adenomatous polyposis coli (APC) protein moves along microtubules and concentrates at their growing ends in epithelial cells. J Cell Biol 148: 505-518. doi:10.1083/jcb.148.3.505

Moissoglu K, Yasuda K, Wang T, Chrisafis G, Mili S. 2019. Translational regulation of protrusion-localized RNAs involves silencing and clustering after transport. Elife 8: e44752. doi:10.7554/eLife .44752

Moissoglu K, Stueland M, Gasparski AN, Wang T, Jenkins LM, Hastings ML, Mili S. 2020. RNA localization and co-translational interactions control RAB13 GTPase function and cell migration. EMBO J 39: e104958. doi:10.15252/embj.2020104958

Mueller F, Senecal A, Tantale K, Marie-Nelly H, Ly N, Collin O, Basyuk E, Bertrand E, Darzacq X, Zimmer C. 2013. FISH-quant: automatic counting of transcripts in 3D FISH images. Nat Methods 10: 277-278. doi:10.1038/nmeth.2406

Munemitsu S, Souza B, Müller O, Albert I, Rubinfeld B, Polakis P. 1994. The APC gene product associates with microtubules in vivo and promotes their assembly in vitro. Cancer Res 54: 3676-3681.

Nakamura M, Zhou XZ, Lu KP. 2001. Critical role for the EB1 and APC interaction in the regulation of microtubule polymerization. Curr Biol 11: 1062-1067. doi:10.1016/S0960-9822(01)00297-4

Nalavadi VC, Griffin LE, Picard-Fraser P, Swanson AM, Takumi T, Bassell GJ. 2012. Regulation of zipcode binding protein 1 transport dynamics in axons by myosin Va. J Neurosci 32: 1513315141. doi:10.1523/JNEUROSCI.2006-12.2012

Oleynikov Y, Singer RH. 2003. Real-time visualization of ZBP1 association with $\beta$-actin mRNA during transcription and localization. Curr Biol 13: 199-207. doi:10.1016/S0960-9822(03)00044-7

Paquin N, Chartrand P. 2008. Local regulation of mRNA translation: new insights from the bud. Trends Cell Biol 18: 105-111. doi:10 .1016/j.tcb.2007.12.004

Poser I, Sarov M, Hutchins JRA, Hériché J-K, Toyoda Y, Pozniakovsky A, Weigl D, Nitzsche A, Hegemann B, Bird AW, et al. 2008. BAC TransgeneOmics. Nat Methods 5: 409-415. doi:10 .1038/nmeth.1199

Preitner N, Quan J, Nowakowski DW, Hancock ML, Shi J, Tcherkezian J, Young-Pearse TL, Flanagan JG. 2014. APC is an RNA-binding protein and its interactome provides a link to neural development and microtubule assembly. Cell 158: 368-382. doi:10.1016/j.cell.2014.05.042

Ronneberger O, Fischer P, Brox T. 2015. U-Net: convolutional networks for biomedical image segmentation. arXiv:150504597 [cs]. http://arxiv.org/abs/1505.04597

Ruane PT, Gumy LF, Bola B, Anderson B, Wozniak MJ, Hoogenraad CC, Allan VJ. 2016. Tumour suppressor adenomatous polyposis coli (APC) localisation is regulated by both Kinesin-1 and Kinesin-2. Sci Rep 6: 27456. doi:10.1038/srep27456

Ryder PV, Fang J, Lerit DA. 2020. Centrocorting RNA localization to centrosomes is regulated by FMRP and facilitates error-free mitosis. J. Cell Biol 219: e202004101. doi:10.1083/jcb.202004101

Siddiqui N, Zwetsloot AJ, Bachmann A, Roth D, Hussain H, Brandt J, Kaverina I, Straube A. 2019. PTPN21 and Hook3 relieve KIF1C autoinhibition and activate intracellular transport. Nat Commun 10: 2693. doi:10.1038/s41467-019-10644-9 
Singer RH. 1993. RNA zipcodes for cytoplasmic addresses. Curr Biol 3: 719-721. doi:10.1016/0960-9822(93)90079-4

Song T, Zheng Y, Wang Y, Katz Z, Liu X, Chen S, Singer RH, Gu W. 2015. Specific interaction of KIF11 with ZBP1 regulates the transport of $\beta$-actin mRNA and cell motility. J Cell Sci 128: 1001-1010.

Stueland M, Wang T, Park HY, Mili S. 2019. RDI calculator: an analysis tool to assess RNA distributions in cells. Sci Rep 9: 8267. doi:10 .1038/s41598-019-44783-2

Tanenbaum ME, Gilbert LA, Qi LS, Weissman JS, Vale RD. 2014. A protein-tagging system for signal amplification in gene expression and fluorescence imaging. Cell 159: 635-646. doi:10.1016/j.cell .2014.09.039

Theisen U, Straube E, Straube A. 2012. Directional persistence of migrating cells requires Kif1C-mediated stabilization of trailing adhesions. Dev Cell 23: 1153-1166. doi:10.1016/j.devcel.2012.11.005

Tsanov N, Samacoits A, Chouaib R, Traboulsi A-M, Gostan T, Weber C, Zimmer C, Zibara K, Walter T, Peter M, et al. 2016. smiFISH and FISH-quant: a flexible single RNA detection ap- proach with super-resolution capability. Nucleic Acids Res 44: e165. doi:10.1093/nar/gkw784

Wang T, Hamilla S, Cam M, Aranda-Espinoza H, Mili S. 2017. Extracellular matrix stiffness and cell contractility control RNA localization to promote cell migration. Nat Commun 8: 896. doi:10 .1038/s41467-017-00884-y

Xing L, Bassell GJ. 2013. mRNA localization: an orchestration of assembly, traffic and synthesis. Traffic 14: 2-14. doi:10.1111/tra .12004

Yasuda K, Clatterbuck-Soper SF, Jackrel ME, Shorter J, Mili S. 2017. FUS inclusions disrupt RNA localization by sequestering kinesin1 and inhibiting microtubule detyrosination. J Cell Biol 216: 1015-1034. doi:10.1083/jcb.201608022

Zumbrunn J, Kinoshita K, Hyman AA, Näthke IS. 2001. Binding of the adenomatous polyposis coli protein to microtubules increases microtubule stability and is regulated by GSK3 $\beta$ phosphorylation. Curr Biol 11: 44-49. doi:10.1016/S0960-9822(01) 00002-1 

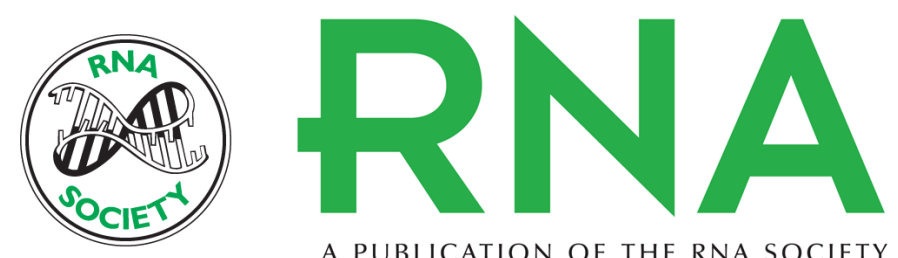

A PUBLICATION OF THE RNA SOCIETY

\section{The kinesin KIF1C transports APC-dependent mRNAs to cell protrusions}

Xavier Pichon, Konstadinos Moissoglu, Emeline Coleno, et al.

RNA 2021 27: 1528-1544 originally published online September 7, 2021

Access the most recent version at doi:10.1261/rna.078576.120

\section{Supplemental http://rnajournal.cshlp.org/content/suppl/2021/09/07/rna.078576.120.DC1 \\ Material}

References This article cites 64 articles, 13 of which can be accessed free at: http://rnajournal.cshlp.org/content/27/12/1528.full.html\#ref-list-1

Open Access Freely available online through the RNA Open Access option.

Creative This article, published in RNA, is available under a Creative Commons License Commons (Attribution 4.0 International), as described at

License http://creativecommons.org/licenses/by/4.0/.

Email Alerting Receive free email alerts when new articles cite this article - sign up in the box at the Service top right corner of the article or click here.

To subscribe to $R N A$ go to:

http://rnajournal.cshlp.org/subscriptions

(C) 2021 Pichon et al.; Published by Cold Spring Harbor Laboratory Press for the RNA Society 